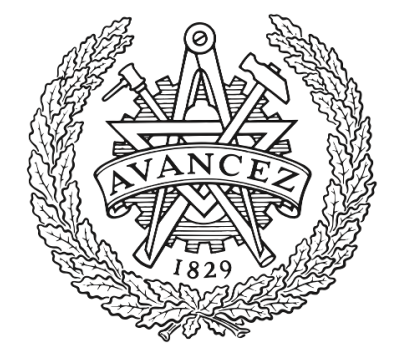

\title{
CHALMERS
}

UNIVERSITY OF TECHNOLOGY

\section{Neutron capture cross sections of light neutron-rich nuclei relevant for -process nucleosynthesis}

Downloaded from: https://research.chalmers.se, 2023-04-26 12:55 UTC

Citation for the original published paper (version of record):

Bhattacharyya, A., Datta, U., Rahaman, A. et al (2021). Neutron capture cross sections of light neutron-rich nuclei relevant for -process nucleosynthesis. Physical Review C, 104(4). http://dx.doi.org/10.1103/PhysRevC.104.045801

N.B. When citing this work, cite the original published paper. 


\title{
Neutron capture cross sections of light neutron-rich nuclei relevant for $r$-process nucleosynthesis
}

A. Bhattacharyya, ${ }^{1,2}$ Ushasi Datta $\odot,{ }^{1,2,3,{ }^{*}}$ A. Rahaman, ${ }^{1,4}$ S. Chakraborty, ${ }^{1,5}$ T. Aumann,,${ }^{3,6}$ S. Beceiro-Novo, ${ }^{7}$ K. Boretzky, ${ }^{3}$ C. Caesar, ${ }^{3}$ B. V. Carlson, ${ }^{8}$ W. N. Catford,${ }^{9}$ M. Chartier, ${ }^{10}$ D. Cortina-Gil, ${ }^{7}$ P. Das,${ }^{1,2}$ G. De. Angelis,${ }^{11}$ P. Diaz Fernandez, ${ }^{7}$ H. Emling, ${ }^{3}$ H. Geissel,,${ }^{3,12}$ D. Gonzalez-Diaz, ${ }^{3}$ M. Heine, ${ }^{3,13}$ H. Johansson, ${ }^{14}$ B. Jonson, ${ }^{14}$ N. Kalantar-Nayestanaki, ${ }^{15}$ T. Kröll, ${ }^{6,16}$ R. Krücken, ${ }^{16}$ J. Kurcewicz, ${ }^{3}$ C. Langer, ${ }^{3}$ T. Le Bleis, ${ }^{16}$ Y. Leifels, ${ }^{3}$ J. Marganiec, ${ }^{17}$ G. Münzenberg, ${ }^{3}$ T. Nilsson, ${ }^{14}$ C. Nociforo, ${ }^{3}$ V. Panin, ${ }^{3}$ S. Paschalis,${ }^{10}$ R. Plag, ${ }^{3}$ R. Reifarth,${ }^{3}$ M. V. Ricciardi, ${ }^{3}$ C. Rigollet, ${ }^{15}$ D. Rossi,, 3 C. Scheidenberger, ${ }^{3,12}$ H. Scheit, ${ }^{6}$ H. Simon, ${ }^{3}$ Y. Togano, ${ }^{17,18}$ S. Typel,,${ }^{3,6}$ Y. Utsuno, ${ }^{19}$

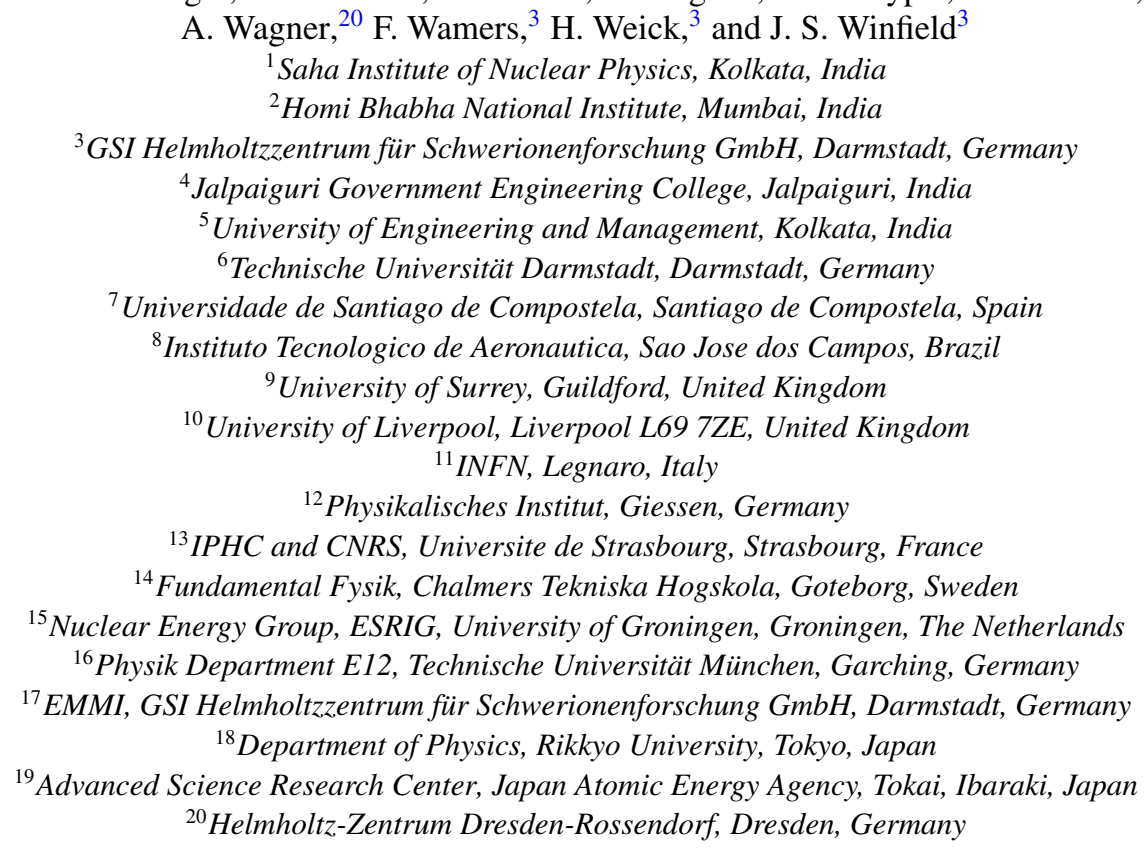

(Received 20 January 2021; revised 13 August 2021; accepted 24 August 2021; published 1 October 2021)

\begin{abstract}
The measurements of neutron capture cross sections of neutron-rich nuclei are challenging but essential for understanding nucleosynthesis and stellar evolution processes in the explosive burning scenario. In the quest of $r$-process abundances, according to the neutrino-driven-wind model, light neutron-rich unstable nuclei may play a significant role as seed nuclei that influence the abundance pattern. Hence, experimental data for neutron capture cross sections of neutron-rich nuclei are needed. Coulomb dissociation of radioactive ion beams at intermediate energy is a powerful indirect method for inferring capture cross section. As a test case for validation of the indirect method, the neutron capture cross section $(n, \gamma)$ for ${ }^{14} \mathrm{C}$ was inferred from the Coulomb dissociation of ${ }^{15} \mathrm{C}$ at intermediate energy $(600 \mathrm{~A} \mathrm{MeV})$. A comparison between different theoretical approaches and experimental results for the reaction is discussed. We report for the first time experimental reaction cross sections of ${ }^{28} \mathrm{Na}(n, \gamma){ }^{29} \mathrm{Na},{ }^{29} \mathrm{Na}(n, \gamma){ }^{30} \mathrm{Na},{ }^{32} \mathrm{Mg}(n, \gamma){ }^{33} \mathrm{Mg}$, and ${ }^{34} \mathrm{Al}(n, \gamma){ }^{35} \mathrm{Al}$. The reaction cross sections were inferred indirectly through Coulomb dissociation of ${ }^{29,30} \mathrm{Na},{ }^{33} \mathrm{Mg}$, and ${ }^{35} \mathrm{Al}$ at incident projectile energies around 400-430 A MeV using the FRS-LAND setup at GSI, Darmstadt. The neutron capture cross sections were obtained from the photoabsorption cross sections with the aid of the detailed balance theorem. The reaction rates for the neutron-rich $\mathrm{Na}, \mathrm{Mg}, \mathrm{Al}$ nuclei at typical $r$-process temperatures were obtained from the measured $(n, \gamma)$ capture cross sections. The measured neutron capture reaction rates of the neutron-rich nuclei, ${ }^{28} \mathrm{Na},{ }^{29} \mathrm{Na}$, and ${ }^{34} \mathrm{Al}$ are significantly lower than those predicted by the Hauser-Feshbach decay model. A similar trend was observed earlier for ${ }^{17} \mathrm{C}$ and ${ }^{19} \mathrm{~N}$ but in the case of ${ }^{14} \mathrm{C}(n, \gamma){ }^{15} \mathrm{C}$ the trend is opposite. The situation is more complicated when the ground state has a multi-particle-hole configuration. For ${ }^{32} \mathrm{Mg}$, the measured cross section is about $40-90 \%$ higher than the Hauser-Feshbach prediction.
\end{abstract}

DOI: 10.1103/PhysRevC.104.045801

*ushasi.dattapramanik@saha.ac.in 


\section{INTRODUCTION}

Understanding the chemical evolution of the universe is a forefront research topic in nuclear astrophysics. The journey began 100 years ago when Arthur Eddington [1] first proposed nucleosynthesis and later, when Fred Hoyle gave a more precise formulation of the nucleosynthesis process. Alpher, Bethe, and Gamow reported the first complete picture of light-elements production in the early universe [2]. A more complete review of the earlier days of nucleosynthesis can be found in [3]. Since then, intense interdisciplinary activities involving state of the art radioactive ion beam research and astronomical observation, as well as astrophysical model calculations with the input of nuclear properties, have advanced our understanding of nucleosynthesis processes. In recent times, in particular, we are witnessing a golden era of understanding of the chemical evolution of the universe. Once-hypothesized neutron star merger events are now an observational fact, after the recent observations by LIGO [4] and the gravitational-wave detection facility VIRGO. A large number of heavy elements, such as platinum, gold, etc., were observed during the neutron star merger event, which provided the first direct evidence of the long sought-after theory of the $r$-process [4]. Recent astronomical observations are witnessing a universal pattern of solar-like $r$-process abundances in old and metal-poor stars. Almost half of the nuclei heavier than iron in the universe are synthesized by rapid $(r-)$ neutron capture processes. More than $90 \%$ of the elements such as europium, gold, and platinum in the solar system are believed to be synthesized by the $r$-process. Initially, neutron capture on heavy nuclei in the $r$-process was proposed to proceed faster than $\beta$ decay when the stellar matter expands and cools again $[5,6]$. Thus the $r$-process produces highly unstable neutronrich nuclei and its path runs close to the neutron-drip line. However, the astronomical site of this nucleosynthesis process has not been determined with certainty. The abundance pattern of $r$-process elements has been suggested to be independent of the metallic content of the progenitor stars [7-10]. A recent calculation [11] shows that neutron star (NS)-neutron star and neutron star-black hole mergers could be the major sources of $r$-process elements in our galaxy. The observed solar like $r$-process pattern in metal poor stars could be due to NS mergers but the rate of occurring NS mergers during the early evolution of the Milky Way would have to be sufficiently large [12], which is again a matter of debate. Recent simulations [13] of the $r$-process for matter ejected dynamically in a NS merger show the influence of $\beta$ and neutron-induced fission and their impact on $r$-process abundance signature peaks. Even some interesting properties of neutron-rich nuclei like pygmy resonances might influence $r$-process abundances [14]. Over the years, detailed astronomical observations, supported by theoretical modeling of different possible sources of high neutron flux, suggest a distinction between a main, a weak and an intermediate $r$-process, each of these associated to a specific type of source [15]. Historically, core-collapse supernovae and neutron star mergers have been recognized as the most promising scenarios for the $r$-process, where the radiative neutron capture reaction of neutron-rich nuclei plays a crucial role and a large number of unstable neutron-rich nuclei are synthesized. Presently, the consensus is that the neutron star mergers may be a site for main $r$-process, while the weak $r$-process may be linked to core-collapse supernovae with neutrino driven winds [16]. One network study was centered on a neutrino-driven wind model [17] for the supernova explosion with a very short dynamical expansion timescale of a few milliseconds. This scenario reduced the production of seed nuclei and enhanced the neutron-to-seed ratio, aiding the synthesis of heavy $r$-process nuclei. The authors found that the introduction of light neutron-rich nuclei to the reaction network changed the abundance of heavy elements by up to an order of magnitude. To understand the influence of light neutron-rich nuclei as seed nuclei in the $r$-process or the synthesis of heavier neutron-rich nuclei, accurate measurement of the $(n, \gamma)$ capture cross sections of neutron-rich $s d p f$ shell nuclei are necessary. Until recently, the only reliable means of obtaining this cross section was to measure it directly in the laboratory with a low energy beam and extrapolate the result to the energies relevant to astrophysical sites. The principal difficulty for measuring radiative capture cross section arises from the very low cross section (of the order of $\mu \mathrm{b}$, $p \mathrm{~b}, n \mathrm{~b})$ and the very low energy beam, requiring the target thickness to be thin considering the energy loss of projectile. In addition, at a number of astrophysical sites, such as nova explosions, explosive supernovae (core-collapse), solar fusion or explosive hydrogen burning, $r$-processes where the temperature is high enough $\left(\approx 10^{8} \mathrm{~K}\right)$, the interaction time can be so short ( $\approx$ seconds) that the unstable nuclei formed in the reaction can undergo subsequent nuclear processes before they decay to stable nuclei. In this scenario, radioactive targets are necessary to study the capture cross sections of unstable nuclei. In particular, the direct measurement of the neutron capture cross section of unstable nuclei with very short lifetimes is extremely difficult and many times impossible. In order to overcome these limitations and difficulties, various indirect methods have been explored in the recent years at various laboratories in the world. These indirect measurements are Coulomb dissociation (CD) [18-20], the ANC method (asymptomatic normalization coefficient) [21,22] and the Trojan-horse method [23,24]. Another popular indirect measurement technique is the surrogate method [25-30]. In this case, a different "surrogate" reaction is considered in which the same compound nucleus as of the desired reaction is formed and its decay products are observed in order to extract the neutron capture cross section. A newer, novel technique, known as the $\beta$-Oslo method [31], is also used for $(n, \gamma)$ cross section estimates with promising results. In this method, a daughter nucleus emits photons, the summed energy of which is measured in coincidence with simultaneous $\beta$ decay of an implanted isotope. Among these methods, most attractive point of the Coulomb dissociation method is the electromagnetic interaction, which is better known than the nuclear interaction and whose parameters are well studied. Moreover, for loosely bound or moderately bound nuclei, the cross section is as larger compared to other methods. It would be interesting to calculate $(n, \gamma)$ reaction rates deduced using the different methods and compare them with the rates 
obtained by the CD method. An intermediate energy relativistic unstable beam can solve the problem of a radioactive target. When the beam energy is high enough, one can use a thick target, which provides a larger yield. The reaction background is also under control in this case. The CD cross section is an order of magnitude larger than the capture cross section due to the phase space factor. Moreover, CD data at one beam energy can provide capture cross sections up to 5-20 MeV in the center of mass energy, depending on the adiabatic cutoff. Since the decay products are kinematically forward focused, a smaller dimension detector can cover a much larger solid angle to detect the reaction products. With this indirect method, one can infer the capture cross section of unbound nuclei which would not be possible in a direct method.

In this paper, we shall present neutron capture cross sections and reaction rates at the temperature of $r$-process sites for neutron-rich nuclei measured by the indirect method of Coulomb dissociation. In the discussion section, we present a very detailed comparison of the ${ }^{14} \mathrm{C}(n, \gamma){ }^{15} \mathrm{C}$ reaction cross section measured by different techniques and methods. The results are also compared to different theoretical predictions. Thus we provide confidence of the validity of the indirect Coulomb dissociation method at intermediate energy. The data for the neutron capture reaction rate on light neutronrich nuclei are also important for understanding $r$-process concerning the neutrino-driven wind model and the impact of light neutron-rich nuclei as seed nuclei. Until recently, $(n, \gamma)$ capture cross sections measured using this method have been reported only for lower $s d$-shell neutron-rich nuclei $[32,33]$. In this work, we shall report the measurements of the neutron capture cross sections for the upper $s d$ and $p f$ shell neutron-rich nuclei, ${ }^{28} \mathrm{Na}(n, \gamma){ }^{29} \mathrm{Na},{ }^{29} \mathrm{Na}(n, \gamma){ }^{30} \mathrm{Na}$, ${ }^{32} \mathrm{Mg}(n, \gamma){ }^{33} \mathrm{Mg}$, and ${ }^{34} \mathrm{Al}(n, \gamma){ }^{35} \mathrm{Al}$ that were considered as seed nuclei in the neutrino-driven wind model. We utilized the indirect CD method to study the time-reversed reactions ${ }^{29} \mathrm{Na}(\gamma, n){ }^{28} \mathrm{Na},{ }^{30} \mathrm{Na}(\gamma, n){ }^{29} \mathrm{Na}$, and ${ }^{33} \mathrm{Mg}(\gamma, n){ }^{32} \mathrm{Mg}$ in inverse kinematics. The CD cross sections were measured through kinematically complete measurements using a ${ }^{208} \mathrm{~Pb}$ target and the FRS-LAND setup at GSI, Darmstadt, Germany. Our analysis follows the procedure given in [18,19,34$36]$, whereby we have obtained the $(n, \gamma)$ capture cross sections versus neutron energy $E_{n}$ from the time reversed $(\gamma, n)$ photoabsorption cross sections. The deduced neutron-capture reaction rates of the neutron-rich nuclei are also compared with a Hauser-Feshbach calculation.

\section{EXPERIMENTAL SETUP}

The experiment was performed at GSI, Darmstadt in Germany, where the isotopes ${ }^{29,30} \mathrm{Na}$ and ${ }^{33} \mathrm{Mg}$ and ${ }^{35} \mathrm{Al}$ were produced by fragmentation of a ${ }^{40} \mathrm{Ar}$ beam with an energy of $540 \mathrm{~A} \mathrm{MeV}$ using the FRS-ALADIN-LAND setup. The fragments were separated by the fragment separator (FRS) [37] and the secondary beams were transported to the experimental site for complete kinematic measurements using ${ }^{208} \mathrm{~Pb},{ }^{12} \mathrm{C}$, and empty secondary targets. Each of the secondary targets was surrounded by $162 \mathrm{NaI}(\mathrm{Tl})$ detectors [38] for detecting $\gamma$ rays emitted from the excited core of the projectiles after
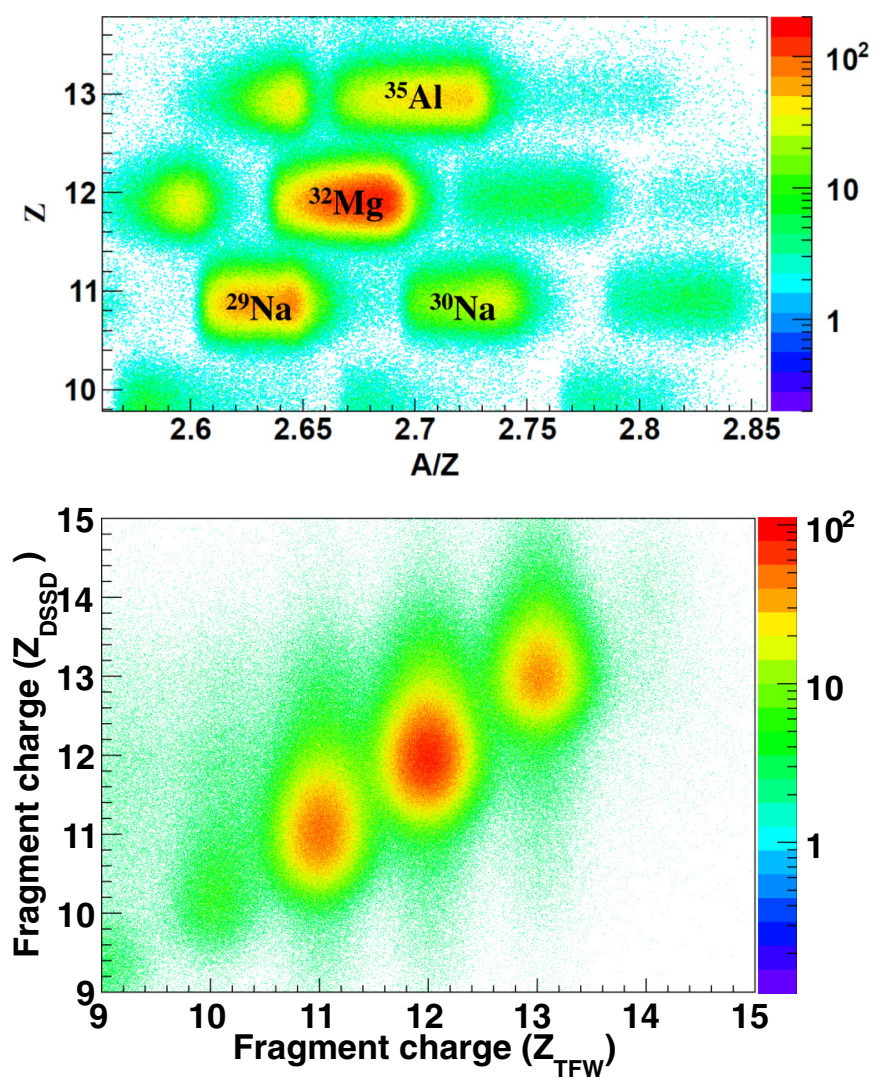

FIG. 1. (Top) Identification plot for a mixed radioactive beam impinging on the secondary targets. (Bottom) Charge distribution of the outgoing fragments. Figure reprinted from [43], copyright by Institute of Physics, 2017.

Coulomb breakup. Depending on their charge-to-momentum ratios, the reaction fragments were deflected at different angles inside ALADIN, which were measured by the GFI detectors $[39,40]$ placed at an angle of $15^{\circ}$ from the original beam direction at two different distances after ALADIN, and were then detected at the time of flight wall detector (TFW). From the energy loss measurements of the TFW and silicon strip detectors (DSSD), the charge distribution of the outgoing fragments was determined. Figure 1 (top) shows the incoming beam identification plot with mass/charge distribution against charge distribution. Figure 1 (bottom) shows the outgoing particle charge identification plot. The neutrons emitted from the decay of the excited projectiles were forward focused and detected by the large area neutron detector (LAND) [41]. From the deflection angles measured by GFI, the time of flight measurements of the reaction fragments, the energy loss at the TFW and the magnetic rigidity of ALADIN, the masses of the outgoing reaction fragments were determined. For the detailed experimental setup and detector calibrations, see [42-44]. A direct breakup model $[19,34,42,45-50]$ was used for the analysis of the experimental data of the nonresonant continuum contribution of the Coulomb excitation. The CD cross section was measured using a $\mathrm{Pb}$ target. However, breakup data were also taken for a carbon target to estimate nuclear contributions of the $\mathrm{Pb}$ target and from an empty target for 
information on background reactions which could take place in various detector materials. The nuclear contribution in the $\mathrm{Pb}$ target was obtained from the $\mathrm{C}$ target data with a scaling factor $[42,48,49]$. The scaling factor was determined using the soft sphere model [51]. Thus, after subtraction of the empty target data and $\mathrm{C}$ target data with normalization, one can obtain a pure $\mathrm{CD}$ differential spectrum. Various components of differential CD cross sections were obtained in coincidence with the corresponding peaks of the summed energy of the $\gamma$-ray spectra. The $\gamma$-ray spectra from reactions with high energy projectiles on high- $Z$ targets are strongly affected by the low-energy (up to energies of a few $\mathrm{MeV}$ ) background. This background originates from atomic interactions of the beam with the lead target $[42,48,49]$. That atomic background spectrum was obtained by considering noninteracting beam condition. Those spectra for the neutron-rich nuclei were shown as a component in the summed energy spectra of the $\gamma$ rays $[42,48,49]$. The summed energy spectrum of $\gamma$ rays was obtained by summing energies of time-correlated $\gamma$ rays events after Doppler correction followed by add-back. The energy resolution is mainly originated from intrinsic resolution of the $\mathrm{NaI}$ crystal and the Doppler effect by the angular opening of the NaI crystal. This resolution is around $15 \%$. The feeding corrections and efficiency correction were calculated using a GEANT4 simulation (Cascade Bertini model) $[42,48,49,52,53]$. For validation of simulation, the simulated results have been compared with source data $\left({ }^{133} \mathrm{Cs},{ }^{60} \mathrm{Co}\right)$ and the results are in agreement with simulation within $10 \%$. To obtain the data for the capture cross section free from experimental constraints, such as statistical fluctuations, detector response, acceptance and efficiency, we fitted the data with the direct breakup model via $\chi^{2}$ minimization and the information on the valence neutron occupation probability in a particular orbital was obtained. In order to facilitate a comparison of calculations within this model with the experimental data, the calculated cross sections were convoluted with the instrumental response. The instrumental response was obtained from Monte Carlo simulation [46,48,54]. The instrumental response for a given value of $E^{*}$ is Gaussian distributed to a good approximation; the width depends on the neutronfragment relative energy $E_{\text {rel }}\left(E^{\star}-S_{n}\right)$ as $\Sigma\left(E_{\text {rel }}\right)=0.04+$ $0.19 \times E_{\mathrm{rel}}^{0.79}\left[\Sigma\left(E_{\mathrm{rel}}\right)\right.$ and $E_{\mathrm{rel}}$ in MeV]. Full geometrical acceptance for one neutron is obtained up to $3.0 \mathrm{MeV}$ relative energy between core and neutron after which a smooth drop is observed due to acceptance. Due to statistical fluctuations in the data, we obtained an error in occupation probability. In addition to that statistical error, a systematic error of 5\% was estimated. Thus, to obtain the photodissociation cross sections, we have used direct breakup (DB) model data for which the valence neutron occupying orbital $(l)$ along with spectroscopic factor were obtained from fitted experimental differential CD cross section and normalized the DB model data with the spectroscopic factor along with statistical error and systematic error. The details of the direct breakup model calculation and the process for obtaining the spectroscopic factors of the valence neutron occupation orbitals were described in previous publications $[34,42,45,46,48,49]$.

The salient features of the analysis procedure and direct breakup model calculation for Coulomb breakup at interme- diate energy are mentioned in the next section. A detailed response of the $\mathrm{NaI}$ detector array for detection of the sumenergy spectrum of the $\gamma$ rays decaying from a particular excited state in the experimental situation, has been generated using the GEANT4 code (Bertini cascade model). In the simulation, the decay scheme of various excited states of ${ }^{28,29} \mathrm{Na}$, ${ }^{34} \mathrm{Al},{ }^{32} \mathrm{Mg}[42,48,49]$ were considered according to the reported values [55]. The partial exclusive Coulomb breakup cross sections of ${ }^{29,30} \mathrm{Na},{ }^{35} \mathrm{Al},{ }^{33} \mathrm{Mg}$ for populating various excited states of ${ }^{28,29} \mathrm{Na},{ }^{34} \mathrm{Al},{ }^{32} \mathrm{Mg}$, respectively, have been extracted from the invariant mass spectra in coincidence with the sum energy of $\gamma$ rays with the different energy ranges. The detection efficiencies and feeding corrections from higher states of $\gamma$ rays have been obtained from detailed simulations. For details see $[42,48,49]$.

\section{COULOMB DISSOCIATION OF UNSTABLE NUCLEI AT INTERMEDIATE ENERGY AND CAPTURE CROSS SECTIONS IN AN ASTROPHYSICAL SCENARIO}

When a projectile moving with high velocity passes a target of high nuclear charge $Z$, it may be excited by absorbing virtual photons from the time-dependent Coulomb field [18]. The projectile may be excited to an unbound state and decays into a core and a nucleon or cluster of nucleons. This CD cross section $\frac{d \sigma}{d E^{*}}$ is related to the photodissociation $A(\gamma, x) B$ cross section, which in turn is related to the inverse reaction $B(x, \gamma) A$ via the principle of detailed balance [18]:

$$
\begin{gathered}
\frac{d \sigma_{c}}{d E^{*}} \rightarrow \sigma^{\gamma}(\gamma, x), \\
\sigma^{\gamma}(\gamma, x) \rightarrow \sigma^{\text {capture }}(x, \gamma) .
\end{gathered}
$$

By measuring the four-momenta of all decay products of the projectile after inelastic scattering followed by breakup, the excitation energy of the nucleus can be determined. The electromagnetic excitation in energetic (several hundreds of $\mathrm{MeV} /$ nucleon) heavy ion collisions is dominated by dipole excitation. The nonresonant direct breakup cross section $d \sigma / d E^{*}$ due to Coulomb interaction can be expressed as

$$
\begin{aligned}
\frac{d \sigma_{c}}{d E^{*}}= & \frac{16 \pi^{3}}{9 \hbar c} n_{E 1}\left(E^{*}\right) \sum_{n l j} C^{2} S\left(I_{c}^{\pi}, n l j\right) \\
& \times \sum_{m}\left|\left\langle q\left|(Z e / A) r Y_{m}^{1}\right| \psi_{n l j}\right\rangle\right|^{2} .
\end{aligned}
$$

$\psi_{n l j}(\vec{r})$ represents the single-particle wave function of the valence neutron in the projectile ground state and $C^{2} S\left(I_{c}^{\pi}, n l j\right)$ its spectroscopic factor with respect to a particular core state $\left(E_{c} ; I_{c}^{\pi}\right)$. The final-state wave function $|q\rangle$ of the valence neutron in the continuum may be approximated by a plane or distorted wave. The distorted wave calculation was performed with an optical potential obtained from systematics. Alternatively, an effective-range approach can be used [56] to calculate the reduced transition probabilities (matrix elements). The single-particle wave functions have been derived from a Woods-Saxon potential. $n_{E 1}$ is the number of equivalent dipole photons. The number of virtual photons of electric dipole $(E 1)$ type for different excitation energies of the 


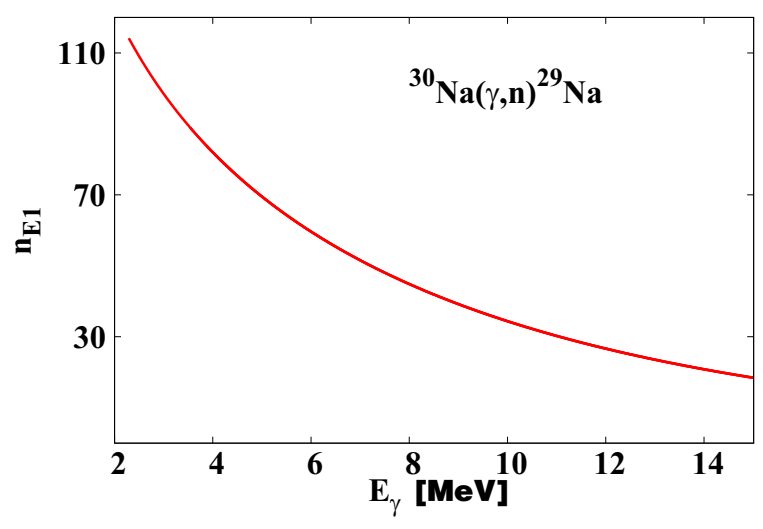

FIG. 2. Variation of number of virtual photons of $E 1$ type with energy $E_{\gamma}$ obtained from an ${ }^{30} \mathrm{Na}$ beam with an energy of $430 A \mathrm{MeV}$ incident on ${ }^{208} \mathrm{~Pb}$ target.

incident nuclei can be obtained from the relation

$$
n_{E 1}=\frac{2}{\pi} Z_{T}^{2} \alpha\left(\frac{c}{v}\right)^{2}\left[\xi K_{0} K_{1}-\frac{v^{2} \xi^{2}}{2 c^{2}}\left(K_{1}^{2}-K_{0}^{2}\right)\right],
$$

where $Z_{T}$ is the target charge, $\alpha$ is the fine structure constant, $v$ is the relative velocity between the projectile and the target, and $K_{0}\left(K_{1}\right)$ is the modified Bessel function of order zero(one). $\xi$ is a dimensionless parameter known as the adiabatic parameter which is defined as

$$
\xi=\frac{w r_{\text {min }}}{\gamma v}
$$

where $w=E^{*} / \hbar$ and $r_{\min }=R_{1}+R_{2}$, where $R_{1}$ and $R_{2}$ are the radii of the projectile and target, respectively. Typical virtual photon spectrum of $E 1$ type for different excitation energies of ${ }^{30} \mathrm{Na}$ at an incident energy of $430 \mathrm{~A} \mathrm{MeV}$ and ${ }^{208} \mathrm{~Pb}$ target is shown in Fig. 2. For measurement of the $(n, \gamma)$ neutron capture cross sections of ${ }^{28,29} \mathrm{Na},{ }^{32} \mathrm{Mg}$, and ${ }^{34} \mathrm{Al}$, relevant in an astrophysical scenario, the indirect $\mathrm{CD}$ method is very useful due to the availability of radioactive neutron rich nuclei at intermediate energy. The ground state configuration and a part of the components of the ground state wave function of ${ }^{29,30} \mathrm{Na},{ }^{33} \mathrm{Mg}$, and ${ }^{35} \mathrm{Al}$ were studied by Coulomb breakup $[42,48,49,52,53]$ using those nuclei as radioactive beams with energies of $400-430 \mathrm{~A} \mathrm{MeV}$ and ${ }^{208} \mathrm{~Pb}$ target. The projectiles were excited to unbound states by absorbing virtual photons from the Coulomb field generated due to their accelerated motion in the vicinity of the target [18]. The valence neutron is excited from the ground state to the continuum through a predominantly $E 1$ type transition [47] and the nucleus breaks into core and a neutron where the core is left as a spectator. Thus, by detecting the neutron in coincidence with different excited states of the fragment, different components of the ground state wave function can be obtained for those nuclei.

After $\mathrm{CD}$ of ${ }^{29,30} \mathrm{Na}[48,52]$ and ${ }^{34} \mathrm{Al}[49,53]$, the core is reported to populate mainly the ground state, with around $30-40 \%$ of the population in the excited states. In the reaction ${ }^{29} \mathrm{Na}$ beam at an energy of $410 \mathrm{~A} \mathrm{MeV}$ was bombarded on a ${ }^{208} \mathrm{~Pb}$ target. The ${ }^{30} \mathrm{Na}$ beam at an energy of $430 \mathrm{~A} \mathrm{MeV}$ was bombarded on a ${ }^{208} \mathrm{~Pb}$ target. A comparison with the direct breakup model suggested that the predominant contribution to the valence neutron occupation in the ground state of ${ }^{29} \mathrm{Na}\left(3 / 2^{+}\right)$and ${ }^{30} \mathrm{Na}\left(2^{+}\right)$was mainly in the $d$ - with a small contribution from the $s$ orbital $[48,52]$. Rahaman et al. $[48,52]$ showed that after the Coulomb breakup of ${ }^{29} \mathrm{Na}$, three excited states of the fragment ${ }^{28} \mathrm{Na}$ were populated, at energies of $1.25 \mathrm{MeV}, 2.12 \mathrm{MeV}$, and $2.71 \mathrm{MeV}$ with spin-parities $\left(2^{+}\right),\left(1^{+}\right)$, and $\left(1^{+}\right)$, respectively, apart from the ground state with a spin-parity of $1^{+}[48,57]$. One of the major components of the ground state configurations for each of these nuclei were ${ }^{28} \mathrm{Na}_{\text {g.s. }}\left(1^{+}\right) \otimes v_{s, d}$ and ${ }^{29} \mathrm{Na}_{\text {g.s. }}\left(3 / 2^{+}\right) \otimes$ $v_{s, d}$, respectively [48]. The ground state spin and parity of these nuclei obtained from the Coulomb breakup measurement were in agreement with previous reported values and the spectroscopic factor for the valence neutron(s) occupying

TABLE I. Spectroscopic factors for the occupied orbitals of the valence neutron(s) in the ground state of ${ }^{29,30} \mathrm{Na}[48],{ }^{33} \mathrm{Mg}[42]$, and ${ }^{35} \mathrm{Al}$ [49] obtained from Coulomb breakup. The asterisk symbols (*) denote spectroscopic factors for the excited states of the cores, ${ }^{28,29} \mathrm{Na},{ }^{32} \mathrm{Mg}$, and ${ }^{34} \mathrm{Al}$ determined in this work.

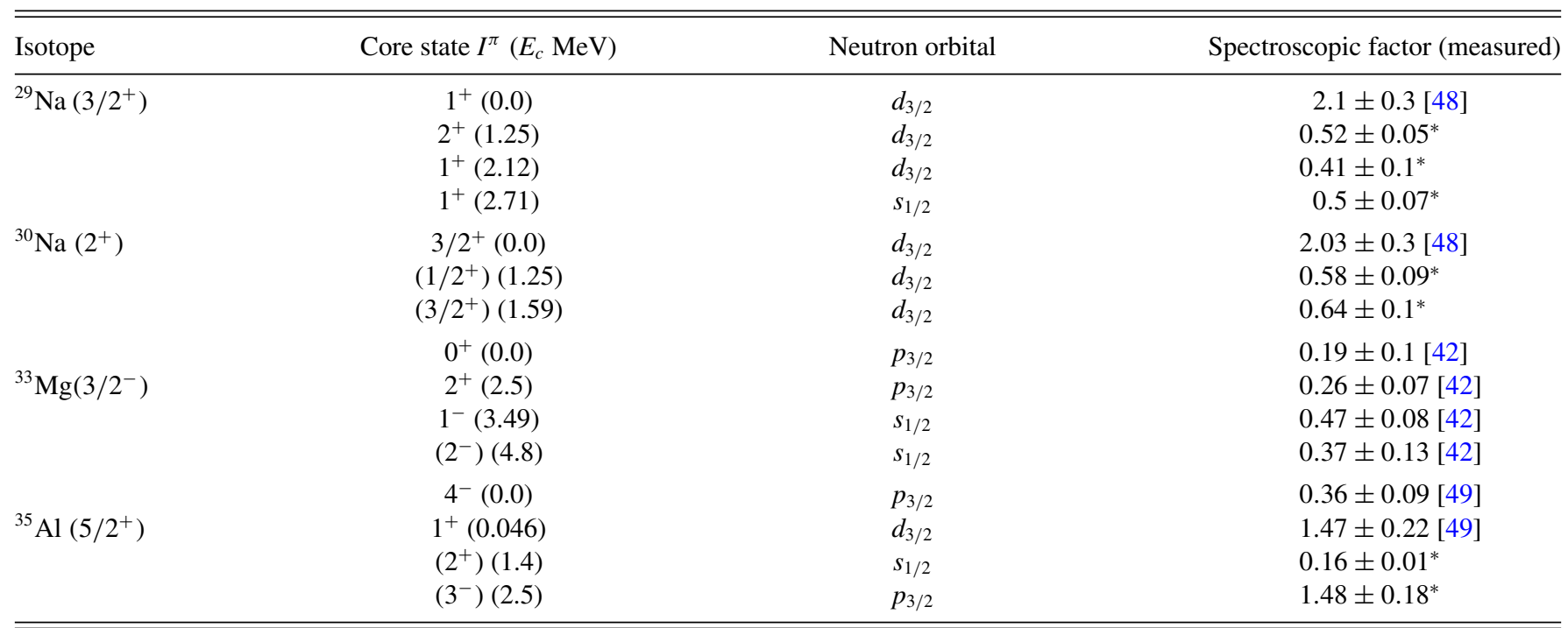



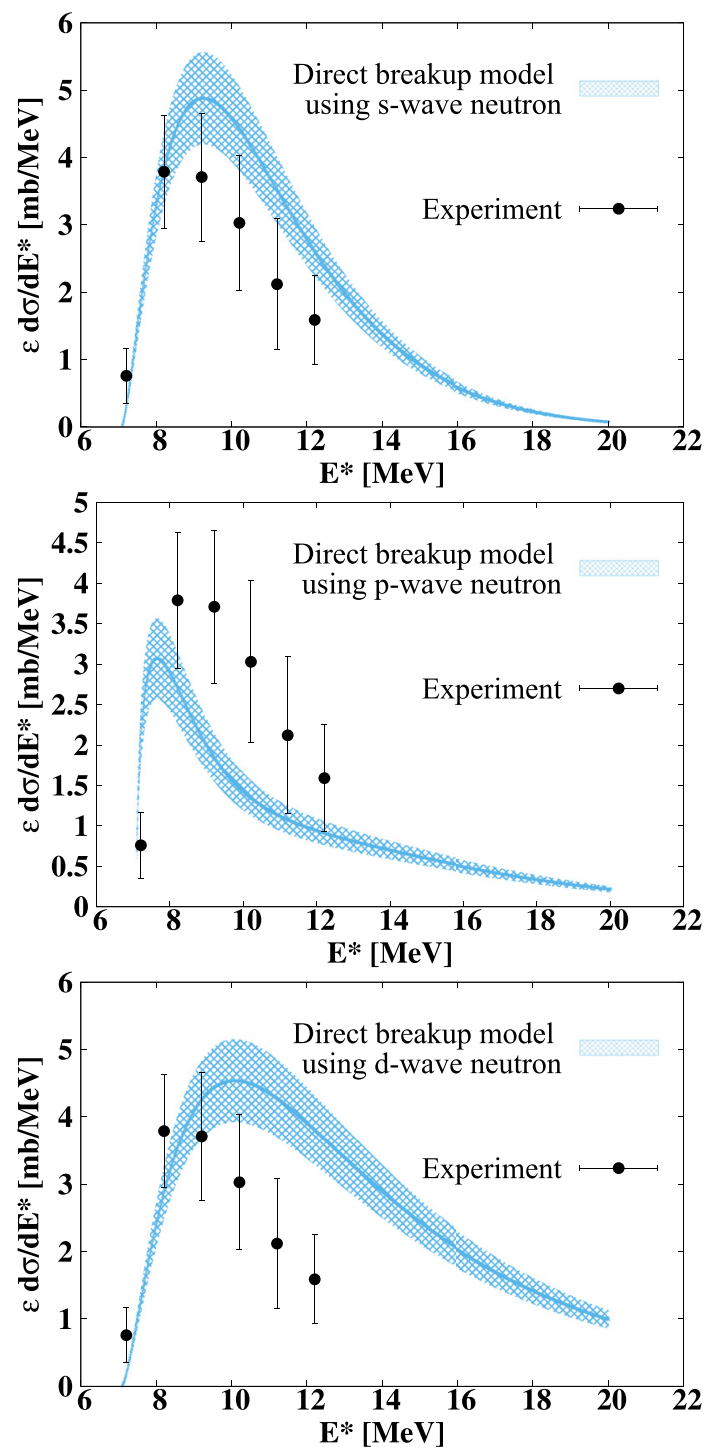

FIG. 3. The data show invariant mass spectrum of ${ }^{29} \mathrm{Na}$ breaking into ${ }^{28} \mathrm{Na}(2.7 \mathrm{MeV})$ and one neutron against excitation energy $\left(E^{*}\right)$. Lines in three panels represent the fitted direct breakup model calculations folded with instrumental response where the valence neutron is occupying the $s$, the $p$ and the $d$ orbital, respectively. The model estimations are normalized with the fitted spectroscopic factors. The shaded regions represent the errors of those.

the $d$ orbital coupled to the core in the ground state was deduced [48]. We have further extended the analysis to obtain other components of the wave function of the ground state of these nuclei. The spectroscopic factors for the valence neutron occupying $d$ orbital which is coupled with the core, ${ }^{28} \mathrm{Na}(1.25$ $\left.\mathrm{MeV}, 2^{+}\right)$and ${ }^{28} \mathrm{Na}\left(2.12 \mathrm{MeV}, 1^{+}\right)$, respectively, have been deduced in the present work. Similarly, as for the $s$ valence neutron orbitals, the coupled with ${ }^{28} \mathrm{Na}\left(2.71 \mathrm{MeV}, 1^{+}\right)$has been obtained, also by comparing the invariant mass spectrum in coincidence with the core state with the direct breakup model calculations via $\chi^{2}$ minimization. The deduced spectroscopic factors for the valence neutron occupying orbitals in the ground state of ${ }^{29} \mathrm{Na}$ are presented in Table I. Figure 3
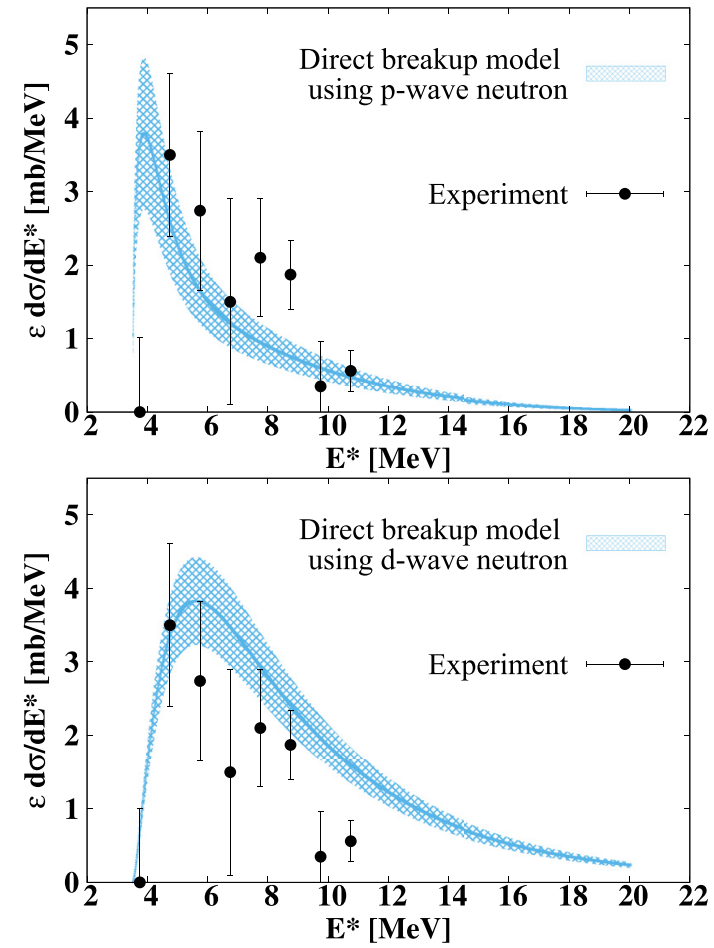

FIG. 4. The data show the invariant mass spectrum of ${ }^{30} \mathrm{Na}$ breaking into ${ }^{29} \mathrm{Na}(1.25 \mathrm{MeV})$ and one neutron against $E^{*}$. The line in the top and bottom panels represents the DB calculation using the $p$ and the $d$ wave for the valence neutron, respectively. The method of fitting was the same as described in Fig. 3.

shows the measured invariant mass spectrum of ${ }^{29} \mathrm{Na}$ breaking into ${ }^{28} \mathrm{Na}(2.71 \mathrm{MeV})$ and one neutron against $E^{*}$. The line in three panels of the figure represents the fitted direct breakup model calculation folded with instrumental response, acceptance, and efficiency where the valence neutron is occupying the $s$, the $p$, the $d$ orbitals, respectively. The calculation was fitted with data via $\chi^{2}$ minimization to obtain spectroscopic factor. The shaded region in Fig. 3 represents the error of the obtained spectroscopic factor. It is clear from the figure that the valence neutron is occupying the $s$ orbital where the obtained $\chi^{2}$ value is minimum. Similarly, the spectroscopic factors for the valence neutron orbitals occupied in the ground state of ${ }^{30} \mathrm{Na}$ have been obtained when the valence neutron is coupled to the core in the ground state [48]. In the present work, the data analyses have been extended and the spectroscopic factors have been deduced when the valence neutron(s) are coupled to the core $\left({ }^{29} \mathrm{Na}\right)$ in the excited states $(1.25$ $\mathrm{MeV}, 3 / 2^{+}$and $1.59 \mathrm{MeV}, 5 / 2^{+}$). The deduced spectroscopic factors for the valence neutron in the ground state of ${ }^{30} \mathrm{Na}$ are presented in Table I. Figure 4 shows measured invariant mass spectrum of ${ }^{30} \mathrm{Na}$ breaking into ${ }^{29} \mathrm{Na}(1.25 \mathrm{MeV})$ and one neutron against $E^{*}$. The line at the top and bottom panels of Fig. 4 represents the fitted direct breakup model calculation where the valence neutron is occupying the $p$ and the $d$ orbital, respectively. The spectroscopic factors has been obtained in a similar procedure as described earlier.

Unlike ${ }^{29} \mathrm{Na}\left(3 / 2^{+}\right)$and ${ }^{30} \mathrm{Na}\left(2^{+}\right)$, for ${ }^{33} \mathrm{Mg}\left(3 / 2^{-}\right)$the major part $\approx(70 \pm 13) \%$ of the cross section was observed 

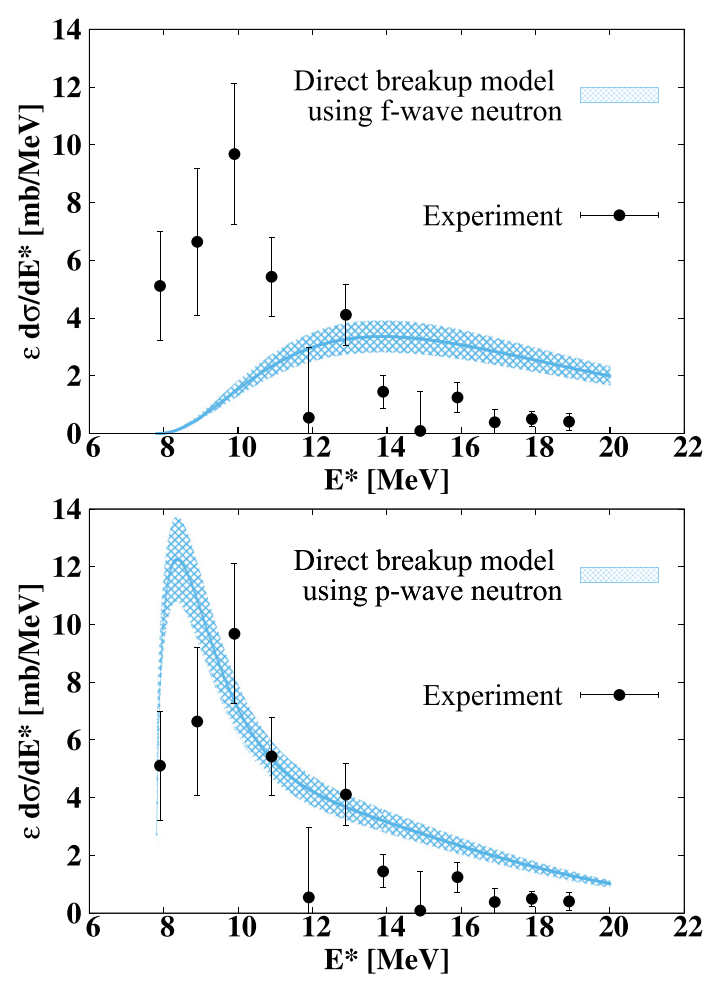

FIG. 5. (Top) The data represent the invariant mass spectrum of ${ }^{35} \mathrm{Al}$ breaking into ${ }^{34} \mathrm{Al}(2.5 \mathrm{MeV})$ and one neutron against $E^{*}$. The line at top and bottom panels shows the DB model predictions where the valence neutron is occupying the $f$ and the $p$ orbital, respectively. The fitting procedure is same as described in Fig. 3.

to populate the excited states of ${ }^{32} \mathrm{Mg}$ after the Coulomb breakup [42]. In that work, it was shown that the shape of the differential CD cross sections in coincidence with different core excited states favor the $s_{1 / 2}$ and the $p_{3 / 2}$ valence neutron orbitals. Those experimental findings suggested a significant reduction and merging of the $s d-p f$ shell gaps at $N \approx 20$ and 28. Thus, the ground state configuration of ${ }^{33} \mathrm{Mg}$ is predominantly a combination of ${ }^{32} \mathrm{Mg}\left(3.0,3.5 \mathrm{MeV} ; 2^{-}, 1^{-}\right) \otimes v_{s_{1 / 2}}$, ${ }^{32} \mathrm{Mg}\left(2.5 \mathrm{MeV} ; 2^{+}\right) \otimes v_{p_{3 / 2}}$, and ${ }^{32} \mathrm{Mg}\left(0 ; 0^{+}\right) \otimes v_{p_{3 / 2}}$, etc. [42]. The deduced spectroscopic factors for the valence neutron(s) occupying orbitals in the ground state of ${ }^{33} \mathrm{Mg}$ [42] are presented in Table I. To deduce the ${ }^{32} \operatorname{Mg}(n, \gamma){ }^{33} \mathrm{Mg}$ capture cross section, we have used the observation mentioned above concerning the ground and excited states of ${ }^{32} \mathrm{Mg}$ that lead to the ground state of ${ }^{33} \mathrm{Mg}$.

The ground state configuration of ${ }^{35} \mathrm{Al}$ was studied by Coulomb breakup [49] and it was shown that after Coulomb breakup, the ground state $\left(4^{-}\right)$and various excited states with energies up to $2.5\left(3^{-}\right) \mathrm{MeV}$ were populated [49,53]. Considering the detector resolution and statistics, the simulated spectrum was fitted with experimental data and several excited states were identified [49]. These states are at energies of $2.5\left(3^{-}\right) \mathrm{MeV}, 1.4\left(2^{+}\right) \mathrm{MeV}$ with an isomeric state at $0.046 \mathrm{MeV}\left(1^{+}\right)$[58]. Chakraborty et al. [49] showed that the principal ground state configuration of ${ }^{35} \mathrm{Al}$ was ${ }^{34} \mathrm{Al}\left(0 ; 4^{-}\right) \otimes v_{p_{3 / 2}}+{ }^{34} \mathrm{Al}\left(0.046 ; 1^{+}\right) \otimes v_{d_{3 / 2}}$ [49]. The deduced spectroscopic factors for the above mentioned con-
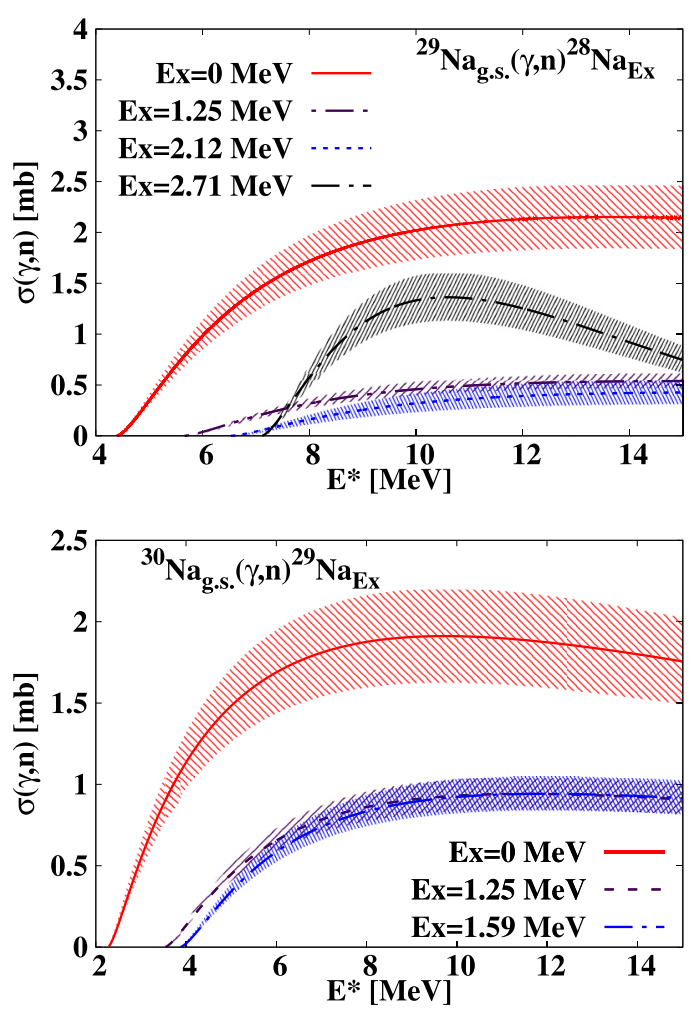

FIG. 6. Variation of photoabsorption cross sections with the excitation energy for ${ }^{29} \mathrm{Na}(\gamma, n){ }^{28} \mathrm{Na}$ (top), ${ }^{30} \mathrm{Na}(\gamma, n){ }^{29} \mathrm{Na}$ (bottom).

figurations [49] are presented in Table I. We further extend the analysis in this work by obtaining other components of the ground state configuration of ${ }^{35} \mathrm{Al}$ and observe that the shape of the differential $\mathrm{CD}$ cross sections in coincidence with the core excited states, $2.5\left(3^{-}\right) \mathrm{MeV}$ and $1.4\left(2^{+}\right) \mathrm{MeV}$, respectively, favor the $p_{3 / 2}$ and the $s_{1 / 2}$ as the valence neutron orbitals. The deduced spectroscopic factors for the valence neutron(s) occupying orbitals which are coupled with excited states are presented in the Table I. Figure 5 shows the measured invariant mass spectrum of ${ }^{35} \mathrm{Al}$ breaking into ${ }^{34} \mathrm{Al}(2.5$ $\mathrm{MeV}$ ) and one neutron against $E^{*}$. The lines in the panels of Fig. 5 represent the fitted DB model calculation folded with instrumental response, as described earlier. The valence neutron is occupying the $f$ and the $p$ orbitals, respectively. As evident from fitted $\chi^{2}$ value, the valence neutron is occupying the $p$ orbital. Thus, all the components of the ground state wave functions of these nuclei have been considered to obtain photoabsorption cross sections. The fits to the direct breakup model CD cross sections were used to obtain the photo-absorption cross sections [18] using the virtual photon numbers and the equation given below:

$$
\frac{d \sigma_{c}}{d E^{*}}=\frac{1}{E^{*}} n_{E 1} \sigma_{E 1}^{\gamma} \text {. }
$$

The fitted direct breakup model calculations have been scaled with the corresponding spectroscopic factors (Table I) for the neutron-rich $\mathrm{Na}, \mathrm{Mg}$, and $\mathrm{Al}$ and the corresponding photoabsorption cross sections have been obtained. Figure 6 (top) and (bottom) shows all the components of the deduced photoabsorption cross sections for ${ }^{29} \mathrm{Na}$ and ${ }^{30} \mathrm{Na}$. Similarly, the 

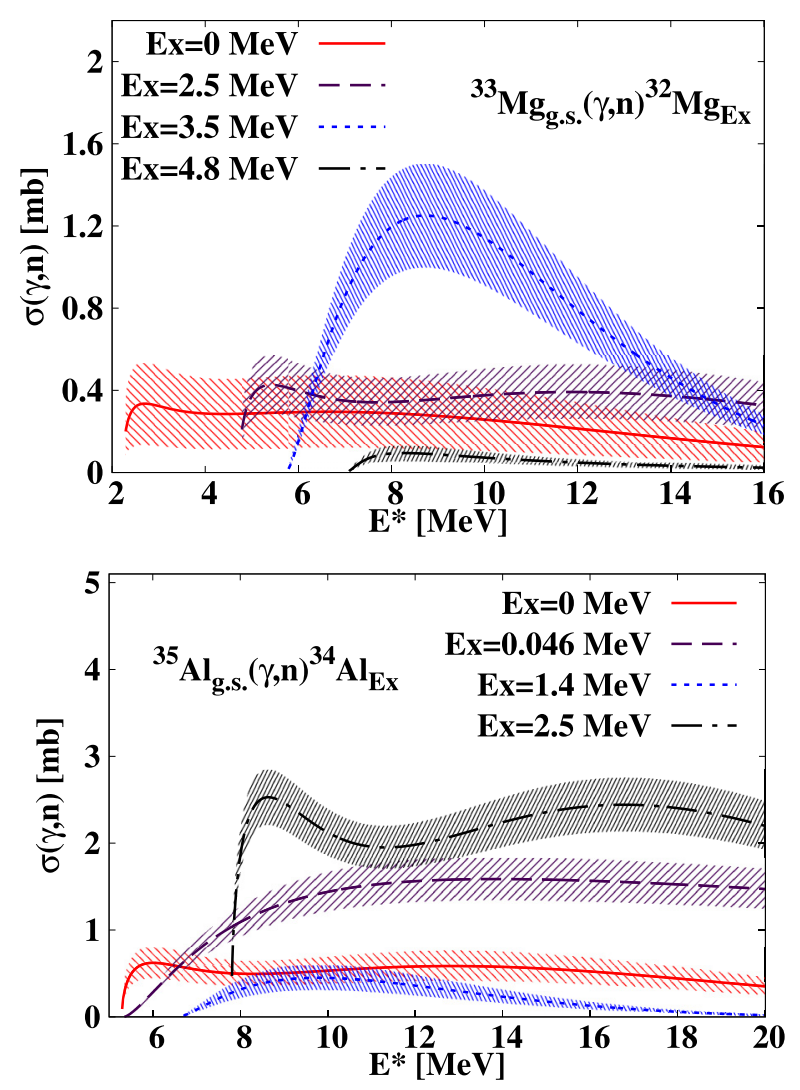

FIG. 7. Photoabsorption cross sections with variation of the excitation energy for ${ }^{33} \mathrm{Mg}(\gamma, n){ }^{32} \mathrm{Mg}$ (top) and ${ }^{35} \mathrm{Al}(\gamma, n){ }^{34} \mathrm{Al}$ (bottom).

different components of the photo-absorption cross sections for ${ }^{33} \mathrm{Mg}$ and ${ }^{35} \mathrm{Al}$ are shown in Fig. 7 (top) and (bottom), respectively. The neutron capture cross sections were obtained from the photoabsorption cross sections using the detailed balance theorem [36]

$$
\sigma_{E 1}^{\text {capture }}=\frac{2\left(2 J_{A}+1\right)}{\left(2 J_{B}+1\right)\left(2 J_{n}+1\right)} \frac{k_{\gamma}^{2}}{k^{2}} \sigma_{E 1}^{\gamma},
$$

where $k_{\gamma}=\frac{E^{*}}{\hbar c}$ and $k^{2}=\frac{2 \mu E_{\text {rel }}}{\hbar^{2}}$ with $\mu$ being the reduced mass of the core and neutron after Coulomb breakup. $J_{A}, J_{B}$, and $J_{n}$ are the spins of the incoming beam, outgoing fragment, and neutron, respectively. The spectra for neutron capture cross sections versus neutron energies $E_{n}$ for ${ }^{28,29} \mathrm{Na}$ and ${ }^{32} \mathrm{Mg},{ }^{34} \mathrm{Al}$ are shown in Figs. 10 and 11, respectively. More details will be given in the discussion section.

\section{ASTROPHYSICAL REACTION RATE}

In astrophysics, the velocity distribution of neutrons in thermal equilibrium with their stellar environment is accounted for by the thermonuclear reaction rate $[59,60]$. It is given in $\mathrm{cm}^{3} \mathrm{~mol}^{-1} \mathrm{~s}^{-1}$ by

$$
N_{A}\langle\sigma v\rangle=N_{A} \sqrt{\frac{8}{\pi \mu\left(k_{B} T\right)^{3}}} \int_{0}^{\infty} d E \sigma(E) E \exp \left(-\frac{E}{k_{B} T}\right),
$$

where $k_{B}$ is the Boltzmann constant, $T$ is the temperature at which the $r$-process occurs, $N_{A}$ is the Avogadro constant, $E$ and $\mu$ refer to the relative energy and the reduced mass of the outgoing fragment and neutron after Coulomb breakup, and $\sigma(E)$ refers to the neutron capture cross section. The total stellar reaction rate $R$ was calculated from the neutron capture reaction rates $R_{i}$ [calculated using Eq. (9)] of the excited states $E_{i}$ with spins $I_{i}$ for the fragments ${ }^{28,29} \mathrm{Na},{ }^{32} \mathrm{Mg}$, and ${ }^{34} \mathrm{Al}$ to the ground state of ${ }^{29,30} \mathrm{Na},{ }^{33} \mathrm{Mg}$, and ${ }^{35} \mathrm{Al}$ using the formula [61]

$$
R=\frac{\sum_{i} g_{i} R_{i} \exp \left(-\frac{E_{i}}{k_{B} T}\right)}{\sum_{i} g_{i} \exp \left(-\frac{E_{i}}{k_{B} T}\right)}
$$

where $g_{i}=2 I_{i}+1$. In the next section, a detailed discussion of the indirect measurement of the ${ }^{14} C(n, \gamma){ }^{15} \mathrm{C}$ capture cross section and reaction rate in a stellar environment will be presented. A detailed comparison between the present measurement and other measurements, as well as various theoretical predictions, will also be presented. New measured results of $(n, \gamma)$ and thermonuclear reaction rates for ${ }^{28,29} \mathrm{Na}$, ${ }^{32} \mathrm{Mg}$, and ${ }^{34} \mathrm{Al}$ will also be presented and will be compared with Hauser-Feshbach calculation.

\section{DISCUSSION}

The reaction, ${ }^{14} \mathrm{C}(n, \gamma){ }^{15} \mathrm{C}$ is important at multiple sites of nucleosynthesis processes. Wiescher et al. reported the possible role of this reaction in the neutron-induced $\mathrm{CNO}$ cycle [62,63] but the first direct measurement [64] at a neutron energy of $23 \mathrm{keV}$ was a factor of five smaller than a direct capture model calculation [65]. It is interesting to note that in AGB stars [66], a relatively large amount of ${ }^{14} \mathrm{C}$ exists on top of the layer of ${ }^{13} \mathrm{C}$ pocket and ${ }^{14} \mathrm{C}(n, \gamma)$ can act as a neutron poison. However, this reaction does not effect model calculations of three solar mass stars [67]. But it would be interesting to check the effect of this reaction in low metallicity stars. That information together with the availability of the radioactive ion beam encouraged us to study the capture cross section measured by Coulomb breakup. Previously, the Coulomb breakup of loosely bound, ${ }^{15} \mathrm{C}$ was studied at an energy 605A MeV [34]. The measured differential Coulomb dissociation cross section of ${ }^{15} \mathrm{C}$ against excitation energy was in good agreement with a direct breakup model calculation. In the calculation it was considered that the valence $s$-wave neutron in the ground state of ${ }^{15} \mathrm{C}$ was excited to a continuum $p$ wave. The valence neutron spectroscopic factor was obtained, which was coupled with ${ }^{14} \mathrm{C}\left(0^{+}\right)$. For the outgoing neutron, both a plane wave and a distorted wave [34] were considered. The interaction between the core and the neutron in the continuum was studied using effective range theory [56]. It is interesting to note that the data of Coulomb breakup of ${ }^{15} \mathrm{C}$ is in good agreement with a plane wave calculation and the calculation with the effective range theory with scattering length parameter of $a_{l}^{1 / 2}=a_{1}^{3 / 2}=-1.37 \mathrm{fm}^{3}$. The values of these two fitted curves are the same (within the error limit, i.e., $5 \%$ ). So the direct capture cross section is also the same. These facts may be interpreted as indicating that the final state of interaction is negligible for ${ }^{15} \mathrm{C}$. The analysis for the neutron 


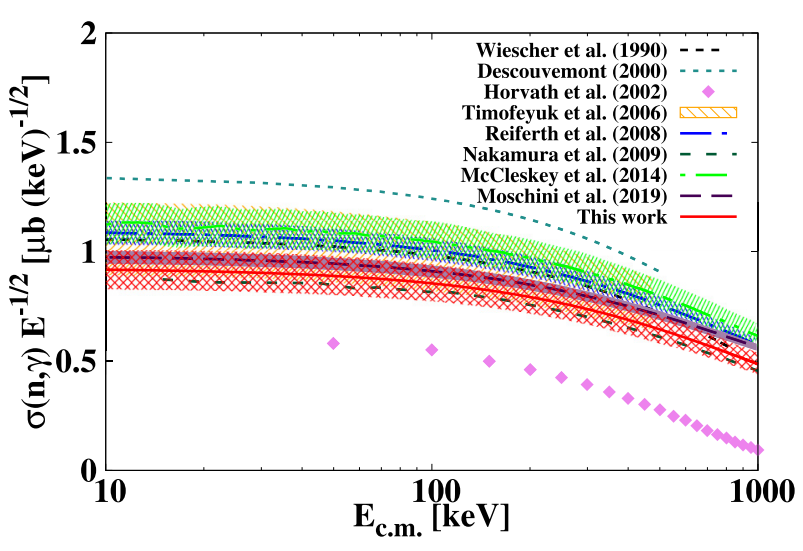

FIG. 8. Plot of $\sigma(n, \gamma) E^{-1 / 2}$ as a function of relative energy between ${ }^{14} \mathrm{C}$ and neutron, $E_{\mathrm{c} . \mathrm{m}}$. obtained from different direct and indirect methods, as well as from calculations.

capture cross section of ${ }^{14} \mathrm{C}(n, \gamma){ }^{15} \mathrm{C}$ was deduced from the fitted direct breakup model calculation using the equations mentioned in the previous section. The estimated error for the present capture cross section data was obtained from the statistical fluctuations of the CD data [34]. To remove the $\sqrt{E}$ energy dependence in the cross section arising from the orbital momentum barrier due to $p$-wave capture, a plot of $\sigma(n, \gamma)$ $E^{-1 / 2}$ was produced (see Fig. 8). A comparison of several available data in the literature along with the present data obtained indirectly from CD data is shown in Fig. 8. The direct neutron capture measurement was performed several times using a radioactive ${ }^{14} \mathrm{C}$ target and a neutron beam at Karlsruhe [68] to improve the accuracy of the measurement and the latest measurement is in agreement with the value obtained from $\mathrm{CD}$ data for ${ }^{15} \mathrm{C}[34,69]$. Our result is in good agreement with the one calculated by Moschini et al. [70] based on a halo effective field theory model and $\mathrm{CD}$ data at lower energy $(60 \mathrm{~A} \mathrm{MeV})$ [20]. The values obtained by the direct neutron capture cross section calculation of Wiescher et al. [65] is also close to present work. The calculation based on charge symmetry in the ${ }^{15} \mathrm{C}$ and ${ }^{15} \mathrm{~F}$ mirror nuclei [71], and cross sections evaluated using the asymptotic normalization coefficients (ANCs) [72] are slightly above the values obtained from the present measurement. A large deviation is observed for the results obtained from microscopic cluster model [73] and from Coulomb breakup at $35 \mathrm{MeV}$ per nucleon [74]. Since the relevant energy for the hot CNO cycle is $23.3 \mathrm{keV}$, the first direct measurement by Beer et al. [64] reported a ${ }^{14} \mathrm{C}(n, \gamma){ }^{15} \mathrm{C}$ cross section at $23.3 \mathrm{keV}$. A value of $4.4 \pm 0.3$ $\mu \mathrm{b}$ is obtained from $\mathrm{CD}$ at that relative energy which is in good agreement with that of [70] but slightly below that of [20]. A comparison of cross sections at $23.3 \mathrm{keV}$ is given in Table II. The reaction rate for neutron capture by ${ }^{14} \mathrm{C}$ in its ground state to the ground state of ${ }^{15} \mathrm{C}$ was determined and compared with HF calculation (see Fig. 9).

Owing to the short-lived nature of the nuclei ${ }^{28} \mathrm{Na}$, ${ }^{29} \mathrm{Na},{ }^{32} \mathrm{Mg}$, and ${ }^{34} \mathrm{Al}$, we had to take recourse to the indirect measurement method of $\mathrm{CD}$ because direct measurement would have been difficult. The components of the ground state wave function of ${ }^{29} \mathrm{Na}$ are ${ }^{28} \mathrm{Na}_{\text {g.s. }}\left(1^{+}\right) \otimes v_{d}$
TABLE II. Comparison of ${ }^{14} \mathrm{C}$ neutron capture cross sections at an energy of $23.3 \mathrm{keV}$, obtained from various measurements and theoretical predictions.

\begin{tabular}{lcc}
\hline \hline Reference & Year & $\sigma(n, \gamma)$ in $\mu \mathrm{b}$ \\
\hline$[64]^{\mathrm{a}}$ & 1992 & $1.72 \pm 0.43$ \\
{$[74]$} & 2002 & $2.6 \pm 0.9$ \\
{$[71]^{\mathrm{b}}$} & 2006 & $5.3 \pm 0.3$ \\
{$[68]$} & 2008 & $5.2 \pm 0.3$ \\
{$[20]^{\mathrm{a}}$} & 2009 & $6.1 \pm 0.5$ \\
{$[72]$} & 2014 & $5.4 \pm 0.5$ \\
{$[70]$} & 2019 & $4.66 \pm 0.14$ \\
This work & & $4.4 \pm 0.3$ \\
\hline \hline
\end{tabular}

adenotes a Maxwellian averaged cross section at $k T=23.3 \mathrm{keV}$.

${ }^{\mathrm{b}}$ denotes cross section at a neutron energy of $23.3 \mathrm{keV}$. Other cross sections were reported at $E_{\mathrm{c} . \mathrm{m} .}=23.3 \mathrm{keV}$

$[48],{ }^{28} \mathrm{Na}\left(2^{+}, 1.25 \mathrm{MeV}\right) \otimes v_{d},{ }^{28} \mathrm{Na}\left(2^{+}, 2.12 \mathrm{MeV}\right) \otimes v_{d}$, and ${ }^{28} \mathrm{Na}\left(2^{+}, 2.71 \mathrm{MeV}\right) \otimes v_{s_{1 / 2}}$, etc. We have deduced the photoneutron cross sections for ${ }^{29} \mathrm{Na}(\gamma, n)^{28} \mathrm{Na}^{*}$ and the neutron capture cross section ${ }^{28} \mathrm{Na}^{*}(n, \gamma){ }^{29} \mathrm{Na}$. Figure 6 (top) shows the photoneutron cross sections of ${ }^{29} \mathrm{Na}(\gamma, n){ }^{28} \mathrm{Na}^{*}$ which populate various states of ${ }^{28} \mathrm{Na}$ while Fig. 10 (top) shows the same or the neutron capture by ${ }^{28} \mathrm{Na}$ in the ground state and the excited states with energies, $E=1.25 \mathrm{MeV}$, $2.12 \mathrm{MeV}, 2.71 \mathrm{MeV}$ and after neutron capture, the reactions populate the ground state of ${ }^{29} \mathrm{Na}$. Figure 12 (top) shows the neutron capture reaction rate for the ${ }^{28} \mathrm{Na}^{*}(n, \gamma){ }^{29} \mathrm{Na}$ reaction for various excited states of ${ }^{28} \mathrm{Na}$ as a function of the temperature $T_{9}$, where $T_{9}=T$ in $\mathrm{K} / 10^{9}$. The reaction rates have been compared with Hauser-Feshbach calculations [75]. Figure 12 (top) shows the comparison between the HauserFeshbach calculation [75] with the measured reaction rates for ${ }^{28} \mathrm{Na}$ in the ground state or in the excited states at 1.25 $\mathrm{MeV}, 2.12 \mathrm{MeV}, 2.71 \mathrm{MeV}$ energies, respectively. Similarly, the extracted components of the ground state wave function of ${ }^{30} \mathrm{Na}$ are ${ }^{29} \mathrm{Na}_{\text {g.s. }}\left(3 / 2^{+}\right) \otimes v_{d}[48],{ }^{29} \mathrm{Na}\left(3 / 2^{+}, 1.25 \mathrm{MeV}\right) \otimes$ $v_{d}$ and ${ }^{29} \mathrm{Na}\left(5 / 2^{+}, 1.59 \mathrm{MeV}\right) \otimes v_{d}$. The photoneutron cross section of ${ }^{30} \mathrm{Na}(\gamma, n){ }^{29} \mathrm{Na}^{*}$ and neutron capture cross section

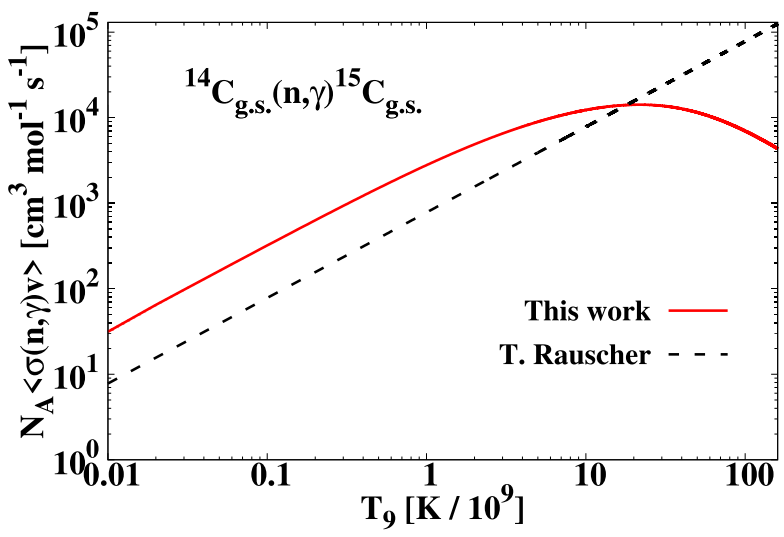

FIG. 9. Variation of reaction rates in $\mathrm{cm}^{3} \mathrm{~mol}^{-1} \mathrm{~s}^{-1}$ with temperature $T_{9}$ for ${ }^{14} \mathrm{C}(n, \gamma){ }^{15} \mathrm{C}$ obtained from both $\mathrm{CD}$ data [34] and Hauser-Feshbach calculation [75]. 

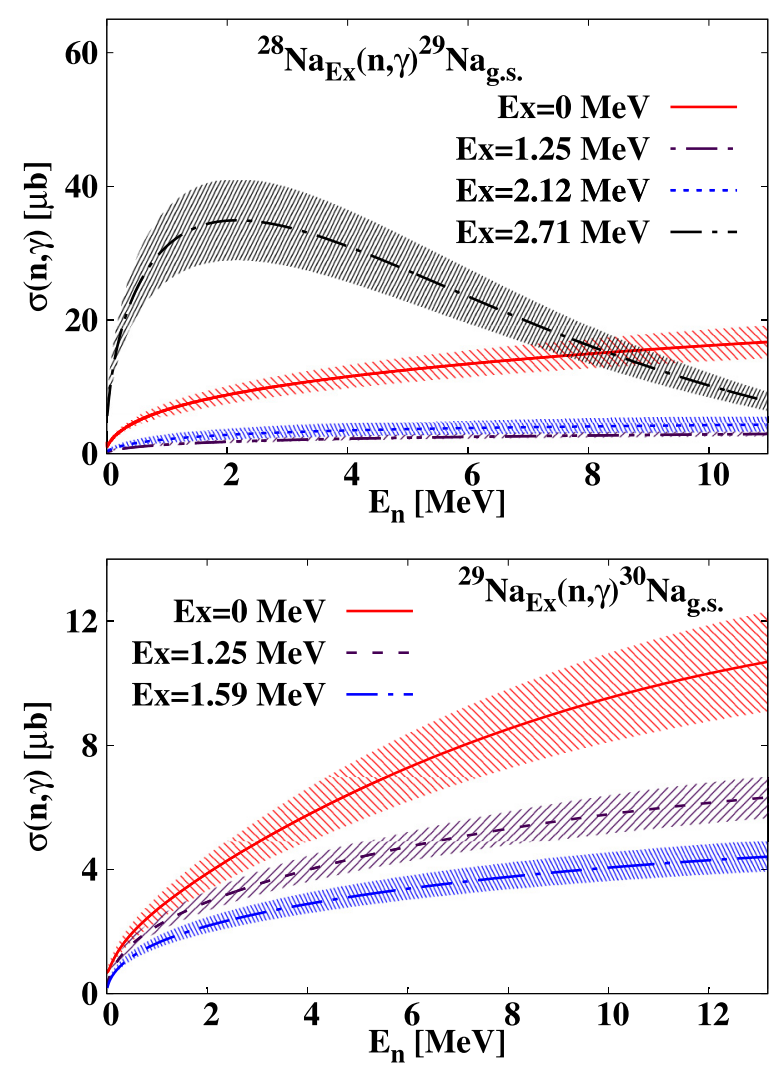

FIG. 10. Neutron capture cross sections as a function of neutron energy $E_{n}$ for ${ }^{28} \mathrm{Na}(n, \gamma){ }^{29} \mathrm{Na}$ (top), ${ }^{29} \mathrm{Na}(n, \gamma){ }^{30} \mathrm{Na}$ (bottom).

of ${ }^{29} \mathrm{Na}^{*}(n, \gamma){ }^{30} \mathrm{Na}$ have been deduced for each component of the ground state wave function for ${ }^{30} \mathrm{Na}$ and compared with Hauser-Feshbach calculations [75]. Figure 6 (bottom) shows all the spectra of the photoneutron cross section of ${ }^{30} \mathrm{Na}(\gamma, n){ }^{29} \mathrm{Na}^{*}$ that populate various states of ${ }^{29} \mathrm{Na}$. It is clear from Fig. 6 that the major part of the photoneutron cross section of ${ }^{29,30} \mathrm{Na}(\gamma, n)$ populates the reaction product in the ground state. Figure 10 (bottom) shows all the spectra of the neutron capture reaction rate for ${ }^{29} \mathrm{Na}^{*}(n, \gamma){ }^{30} \mathrm{Na}$ reaction for various ${ }^{29} \mathrm{Na}$ ground and excited states. The reaction rates were compared with a Hauser-Feshbach (HF) calculation [75]. In Fig. 12 (bottom), shows the HF calculation by Rauscher [75] and various components of the inferred reaction rate obtained from $\mathrm{CD}$ data when ${ }^{29} \mathrm{Na}$ is in its ground state or its excited states at $\left(3 / 2^{+}\right) 1.25 \mathrm{MeV},\left(5 / 2^{+}\right) 1.59 \mathrm{MeV}$ energies, respectively.

The ground state configuration of ${ }^{33} \mathrm{Mg}$ was studied by the $\mathrm{CD}$ method [42] and was reported to be predominantly a combination of ${ }^{32} \mathrm{Mg}\left(3.0,3.5 \mathrm{MeV} ; 2^{-}, 1^{-}\right) \otimes v_{s_{1 / 2}}$, ${ }^{32} \operatorname{Mg}\left(2.5 \mathrm{MeV} ; 2^{+}\right) \otimes v_{p_{3 / 2}}$, and ${ }^{32} \operatorname{Mg}\left(0 ; 0^{+}\right) \otimes v_{p_{3 / 2}}$, etc. [42]. Considering the components of the ground state wave function, the photoneutron cross section of ${ }^{33} \mathrm{Mg}(\gamma, n)^{32} \mathrm{Mg}^{*}$ and the neutron capture cross section of ${ }^{32} \mathrm{Mg}^{*}(n, \gamma){ }^{33} \mathrm{Mg}$ have been deduced and compared with a Hauser-Feshbach calculation [75]. Figure 7 (top) shows the photoneutron cross sections of ${ }^{33} \operatorname{Mg}(\gamma, n){ }^{32} \mathrm{Mg}^{*}$ that populate various excited states of ${ }^{32} \mathrm{Mg}$. The solid line represents the cross section that populates ${ }^{32} \mathrm{Mg}$ in its ground state and excited states at
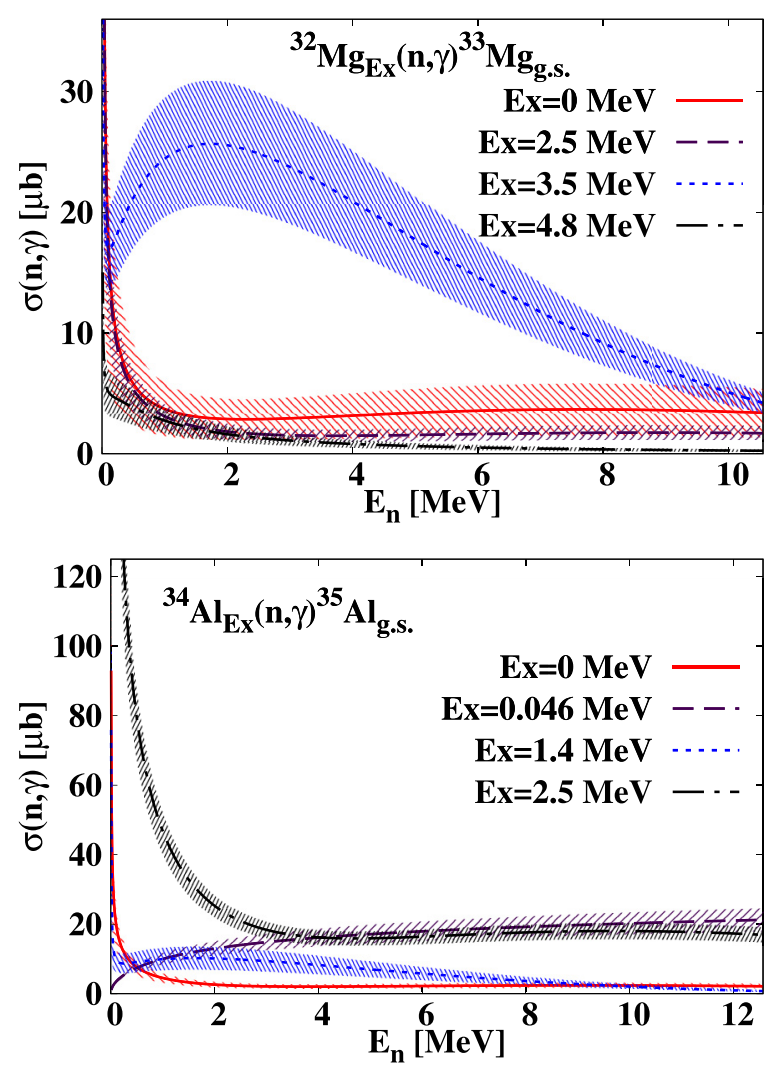

FIG. 11. Variation of the neutron capture cross sections with neutron energy $E_{n}$ for ${ }^{32} \mathrm{Mg}(n, \gamma){ }^{33} \mathrm{Mg}$ (top) and ${ }^{34} \mathrm{Al}(n, \gamma){ }^{35} \mathrm{Al}$ (bottom).

(3.0, 3.5 MeV; $\left.2^{-}, 1^{-}\right),\left(2.5 \mathrm{MeV} ; 2^{+}\right)$, and $\left(4.8 \mathrm{MeV} ; 2^{-}\right)$. It is clear from the figure that the dominant channels for the photoneutron cross sections of ${ }^{33} \mathrm{Mg}$ populate ${ }^{32} \mathrm{Mg}(3.0,3.5$ $\mathrm{MeV} ; 2^{-}, 1^{-}$) and yield a $p$ wave neutron. Figure 11 (top) shows various components of the neutron capture reaction rates for ${ }^{32} \mathrm{Mg}^{*}$ that populate the ground state of ${ }^{33} \mathrm{Mg}$. Figure 13 (top) shows all the measured neutron capture reaction rates for ${ }^{32} \mathrm{Mg}^{*}$ along with $\mathrm{HF}$ estimates [75] as a function of temperature $T_{9}$.

Chakraborty et al. [49] showed that the principal ground state configuration of ${ }^{35} \mathrm{Al}$ is ${ }^{34} \mathrm{Al}\left(0 ; 4^{-}\right) \otimes$ $v_{p_{3 / 2}}+{ }^{34} \mathrm{Al}\left(0.046 \mathrm{MeV} ; 1^{+}\right) \otimes v_{d_{3 / 2}} \quad$ [49]. In the present work, two other components ${ }^{34} \mathrm{Al}\left(1.4 \mathrm{MeV} ; 1^{+}\right) \otimes v_{s_{3 / 2}}+$ ${ }^{34} \mathrm{Al}\left(2.5 \mathrm{MeV} ; 3^{-}\right) \otimes v_{p_{3 / 2}}$ have been extracted. The photoneutron cross sections have been obtained for ${ }^{35} \mathrm{Al}$ from the $\mathrm{CD}$ cross sections related to those components of the ground state wave function. Figure 7 (bottom) shows the variation of the photoneutron cross sections of ${ }^{35} \mathrm{Al}(\gamma, n){ }^{34} \mathrm{Al}^{*}$ with temperature $\left(T_{9}\right)$. It is very clear from the figure that the enhanced photoneutron cross section of ${ }^{35} \mathrm{Al}$ populates the ${ }^{34} \mathrm{Al}\left(2.5 \mathrm{MeV} ; 3^{-}\right)$state and yields a $p$ wave neutron. The neutron capture reaction rates for the ground state and various excited states of ${ }^{34} \mathrm{Al}$ as a function of the temperature $T_{9}$ are shown in Fig. 13 (bottom). The measured reaction rates were compared to a parametrization of Hauser-Feshbach estimates [75]. 

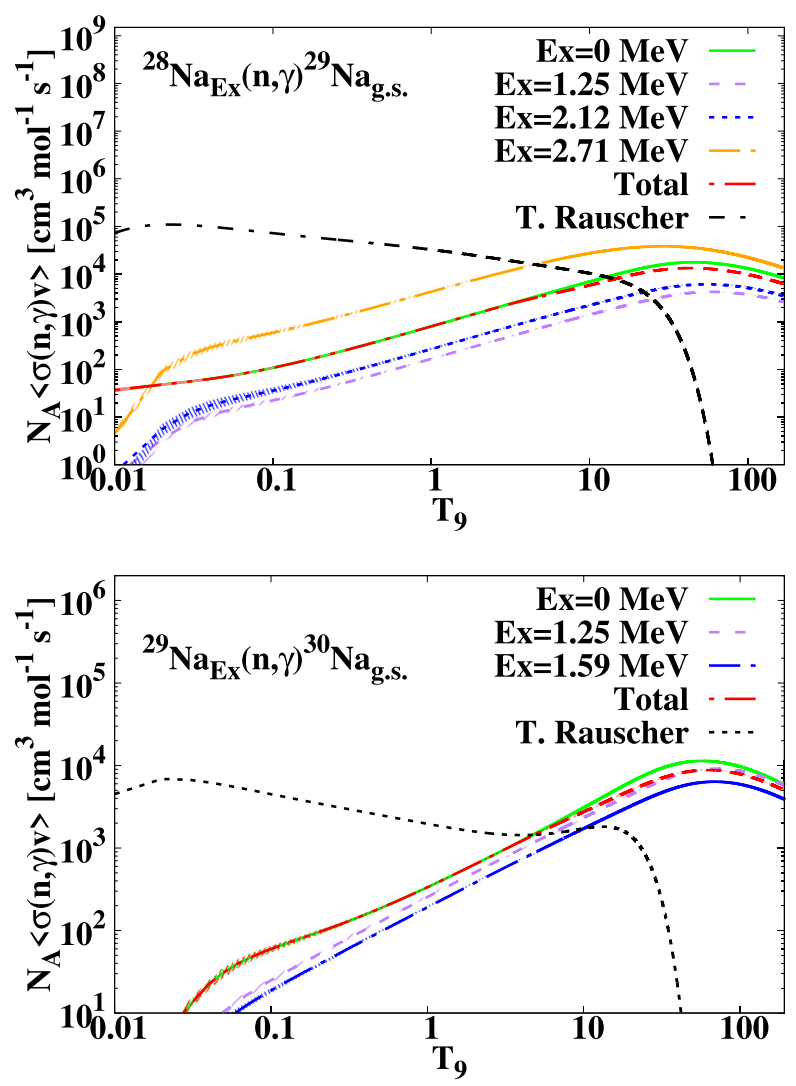

FIG. 12. Variation of reaction rates in $\mathrm{cm}^{3} \mathrm{~mol}^{-1} \mathrm{~s}^{-1}$ with temperature $T_{9}$ for ${ }^{28} \mathrm{Na}(n, \gamma){ }^{29} \mathrm{Na}$ (top), ${ }^{29} \mathrm{Na}(n, \gamma){ }^{30} \mathrm{Na}$ (bottom). The measured reaction rates (represented by the shaded region) have been compared with Hauser-Feshbach estimates (represented by a dot-dashed line) [75].

The measured reaction rates at the onset and end of the $r$-process for the nuclear reactions considered are displayed in Table III. The Hauser-Feshbach rates at these temperatures are also shown. It is evident from Fig. 12 (top) and (bottom) that the indirect measurement of $(n, \gamma)$ reaction rates for ${ }^{28,29} \mathrm{Na}$ are significantly lower (several order of magnitude) compared to Hauser-Feshbach calculations [75] at $r$-process temperatures $0.62 \leqslant T_{9} \leqslant 2$ [17]. This difference is greater for loosely bound neutron-rich nucleus ${ }^{29} \mathrm{Na}(n, \gamma)$ than that for ${ }^{28} \mathrm{Na}(n, \gamma)$. However, if one considers neutron capture reaction rates for excited states of ${ }^{28} \mathrm{Na}$ and, which can populate the ground state of ${ }^{29} \mathrm{Na}$ and ${ }^{30} \mathrm{Na}$, respectively, then the difference between the measured rates and the Hauser-Feshbach estimates [75] are reduced, although the difference is still an order of magnitude. Unlike, ${ }^{28,29} \mathrm{Na}$, the reaction rate for ${ }^{32} \operatorname{Mg}(n, \gamma)$ at $r$-process temperature is in close agreement with HF predictions. But the scenario is different for the ${ }^{34} \mathrm{Al}(n, \gamma)$ reaction rate at $r$-process temperatures. If one considers only neutron capture of ${ }^{34} \mathrm{Al}$ in the ground state, or the first or second excited states, then the measured thermonuclear reaction rate is an order of magnitude lower when compared to the HF prediction [see Fig. 13 (bottom)]. However, the measured thermonuclear reaction rate for neutron capture by excited ${ }^{34} \mathrm{Al}$ close to the
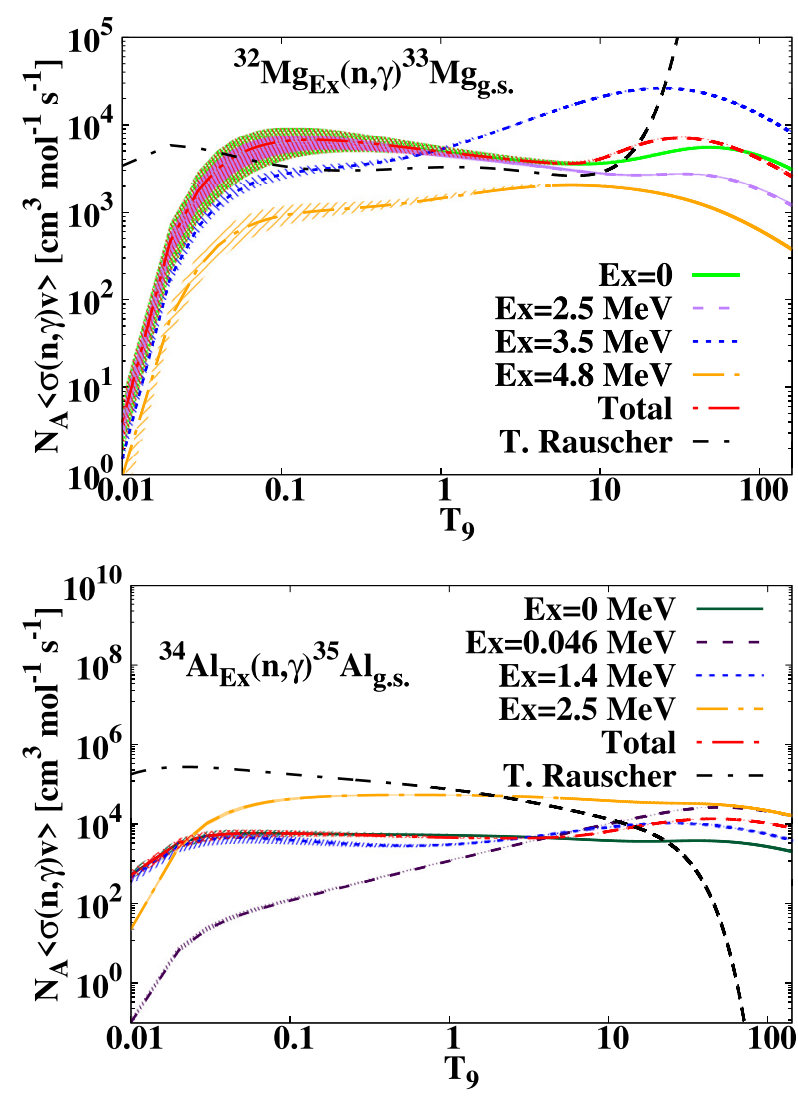

FIG. 13. Variation of reaction rates in $\mathrm{cm}^{3} \mathrm{~mol}^{-1} \mathrm{~s}^{-1}$ with temperature $T_{9}$ for ${ }^{32} \mathrm{Mg}(n, \gamma){ }^{33} \mathrm{Mg}$ (top) and ${ }^{34} \mathrm{Al}(n, \gamma){ }^{35} \mathrm{Al}$ (bottom). The measured reaction rates (shaded region) have been compared with Hauser-Feshbach estimates dot-dashed line) [75].

neutron threshold is closer to the HF prediction [75] [see Fig. 13 (bottom)]. Thus, it is clear that the measured neutron capture reaction rates for ${ }^{28} \mathrm{Na},{ }^{29} \mathrm{Na}$ are lower than the HF predictions [75]. In contradiction, the trend is opposite, when loosely bound valence neutron is occupying low- $l$ orbital like, ${ }^{14} \mathrm{C}(n, \gamma)$ (see Fig. 9). But the situation is different for the nuclei where the ground state configurations are multi-particle-hole. In certain situations, (the ${ }^{34} \mathrm{Al}$ threshold state here), the neutron capture reaction rate by the excited nucleus is enhanced compared to others. Similarly, the measured $p$-wave neutron capture reaction rate by ${ }^{32} \mathrm{Mg}(3.0$, 3.5 MeV; $2^{-}, 1^{-}$) has also been observed to be enhanced. Since the $r$-process is a fast process of the neutron capture, the capture by excited nuclei may play an important role and it would be interesting to include those reaction rates in the $r$-process network calculations for obtaining the abundance curve. Thus, the inferred neutron capture cross section from measured CD data can be useful for understanding impact of the seed nuclei in $r$-process network calculations. On the other hand in the explosive burning scenario, nucleosynthesis process involves the unstable nuclei and capture reaction rates of those unstable nuclei are necessary for astrophysical model calculation. But as it is observed from present studies, HF prediction for unstable nuclei are not always reliable for the exotic nuclei. This can be understood with a simplified 
TABLE III. Capture cross sections and reaction rates for ${ }^{28} \mathrm{Na}(n, \gamma){ }^{29} \mathrm{Na},{ }^{29} \mathrm{Na}(n, \gamma){ }^{30} \mathrm{Na},{ }^{32} \mathrm{Mg}(n, \gamma){ }^{33} \mathrm{Mg}$, and ${ }^{34} \mathrm{Al}(n, \gamma){ }^{35} \mathrm{Al}$ at $r$ process temperatures $0.62 \leqslant T_{9} \leqslant 2$ [17] and corresponding relative energies $53 \mathrm{keV} \leqslant E_{\text {rel }} \leqslant 172 \mathrm{keV}$. The measured reaction rates have been compared with Hauser-Feshbach (HF) estimates [75]. The total cross sections are calculated by summing the cross sections of the ground and excited states and the total reaction rates are calculated using Eq. (9).

\begin{tabular}{|c|c|c|c|c|c|c|c|}
\hline Reaction & $\begin{array}{c}\text { Core state } \\
E_{c} \mathrm{MeV}\end{array}$ & $\sigma(n, \gamma) \mu \mathrm{b}$ & \multicolumn{2}{|c|}{$N_{A}\langle\sigma v\rangle \mathrm{cm}^{3} \mathrm{~mol}^{-1} \mathrm{~s}^{-1}$} & $\sigma(n, \gamma) \mu \mathrm{b}$ & \multicolumn{2}{|c|}{$\mathrm{N}_{A}\langle\sigma v\rangle \mathrm{cm}^{3} \mathrm{~mol}^{-1} \mathrm{~s}^{-1}$} \\
\hline \multirow[t]{4}{*}{${ }^{28} \mathrm{Na}(n, \gamma)$} & 0.0 & $3.01 \pm 0.44$ & $1549.04 \pm 7.92$ & & $1.78 \pm 0.26$ & $510.11 \pm 4.67$ & \\
\hline & 1.25 & $0.63 \pm 0.08$ & $321.77 \pm 4.89$ & & $0.37 \pm 0.05$ & $106.34 \pm 2.89$ & \\
\hline & 2.12 & $1.00 \pm 0.27$ & $515.09 \pm 15.71$ & & $0.60 \pm 0.16$ & $170.48 \pm 9.29$ & \\
\hline & Total & $20.80 \pm 2.83$ & $1547.59 \pm 7.91$ & 23967.14 & $12.5 \pm 1.71$ & $510.11 \pm 4.67$ & 39167.15 \\
\hline \multirow[t]{4}{*}{${ }^{29} \mathrm{Na}(n, \gamma)$} & 0.0 & $1.23 \pm 0.18$ & $630.40 \pm 10.49$ & & $0.82 \pm 0.12$ & $221.55 \pm 6.68$ & \\
\hline & 1.25 & $0.97 \pm 0.10$ & $501.88 \pm 5.76$ & & $0.54 \pm 0.05$ & $158.66 \pm 3.26$ & \\
\hline & 1.59 & $0.72 \pm 0.08$ & $375.88 \pm 4.69$ & & $0.41 \pm 0.04$ & $119.07 \pm 2.66$ & \\
\hline & Total & $2.92 \pm 0.22$ & $630.27 \pm 10.48$ & 1584.51 & $1.77 \pm 0.14$ & $221.55 \pm 6.68$ & 2323.04 \\
\hline${ }^{32} \operatorname{Mg}(n, \gamma)$ & 0.0 & $11.82 \pm 6.96$ & $4270.17 \pm 370.38$ & & $27.88 \pm 16.41$ & $5491.24 \pm 892.34$ & \\
\hline \multirow[t]{5}{*}{${ }^{34} \mathrm{Al}(n, \gamma)$} & 0.0 & $14.66 \pm 4.19$ & $4982.98 \pm 200.51$ & & $27.97 \pm 8.00$ & $5394.38 \pm 380.26$ & \\
\hline & 0.046 & $4.53 \pm 0.70$ & $2329.88 \pm 40.01$ & & $2.54 \pm 0.39$ & $743.25 \pm 22.80$ & \\
\hline & 1.4 & $8.81 \pm 2.81$ & $3785.99 \pm 142.46$ & & $12.48 \pm 3.98$ & $2823.82 \pm 214.71$ & \\
\hline & 2.5 & $107.30 \pm 12.84$ & $51444.89 \pm 909.53$ & & $288.73 \pm 36.20$ & $54355.02 \pm 1704.76$ & \\
\hline & Total & $135.3 \pm 13.81$ & $4443.65 \pm 159.93$ & 50415.94 & $331.72 \pm 37.29$ & $4819.91 \pm 333.31$ & 93557.84 \\
\hline
\end{tabular}

vision by considering the fact that the density of states for the loosely bound neutron-rich nuclei are different than the normal nuclei. So it is expected that HF calculation may not be suitable and a better theoretical approach is necessary. The inferred neutron capture cross sections for neutron-rich nuclei may also play a crucial role for validation of modern theoretical model predictions.

\section{SUMMARY}

The $r$-process conditions were assumed to correspond to a neutrino-driven wind model of a core-collapse supernovae. The neutrino-driven wind model has predicted that the neutron capture reaction of light neutron-rich nuclei may influence r-process abundances when those are considered as seed nuclei. For this purpose, we inferred the neutron capture cross sections of ${ }^{14} \mathrm{C},{ }^{28,29} \mathrm{Na},{ }^{32} \mathrm{Mg}$ and ${ }^{34} \mathrm{Al}$ from measured data of Coulomb dissociation at relativistic energies. We obtained the neutron capture cross sections from the photoabsorption cross sections of ${ }^{29,30} \mathrm{Na},{ }^{33} \mathrm{Mg}$, and ${ }^{35} \mathrm{Al}$ which were used to determine the corresponding neutron capture reaction rates at typical $r$-process temperatures for nucleosynthesis. The ${ }^{14} \mathrm{C}(n, \gamma)$ reaction has been extensively studied by direct and indirect measurements and has also been predicted by different theoretical approaches. We have compared the neutron capture reaction rate obtained from present studies with all calculated and measured data using different approaches. This comparison shows that the neutron capture cross section obtained indirectly from CD data is in closer agreement with that obtained from other $\mathrm{CD}$ measurements at lower energy
$(60 \mathrm{~A} \mathrm{MeV})$, as well as ANC measurement and direct method measurements. The inferred reaction rates ${ }^{14} \mathrm{C}(n, \gamma)$ from $\mathrm{CD}$ data is significantly higher than the Hauser-Feshbach rates but theoretical approach provided by Moschini et al. [70] is in good agreement. The inferred reaction rates for neutron capture on ${ }^{28,29} \mathrm{Na}$ at the relevant $r$-process temperatures are significantly lower (an order of magnitude lower) than those predicted by the Hauser-Feshbach calculation. Previous reports on the indirectly inferred capture rates for ${ }^{16} \mathrm{C}$ and ${ }^{18} \mathrm{~N}$ [32,33] were also significantly lower than the Hauser-Feshbach estimates at such temperatures. However, the situation is different for ${ }^{32} \mathrm{Mg}$ and ${ }^{34} \mathrm{Al}$, for which the neutron capture reaction rates are close to the HF prediction. Thus for a better understanding on the impact of seed nuclei in $r$-process network calculations, a combined effort in experimental and theoretical investigations is necessary.

\section{ACKNOWLEDGMENTS}

U.D. acknowledges the Alexander von Humboldt Foundation, Germany and SEND project grants (PIN:11-R\&D-SIN5.11-0400) from the Department of Atomic Energy (DAE), Government of India for financial support for this work. A.B. and P.D. acknowledge with thanks the financial support provided by CSIR vide file no. 09/489(0115)/2019-EMR-I and 09/489(0111)/2019-EMR-I, respectively. B.V.C. acknowledges support from Grant No. 2017/05660-0 of the São Paulo Research Foundation (FAPESP) and the INCT-FNA Project No. 464898/2014-5. 
[1] A. S. Eddington, Nature (London) 106, 2653 (1920).

[2] R. A. Alpha, H. Bethe, and G. Gamow, Phys. Rev. 73, 803 (1948).

[3] E. M. Burbidge, G. R. Burbidge, W. A. Fowler, and F. Hoyle, Rev. Mod. Phys. 29, 547 (1957).

[4] B. P. Abbott et al. (LIGO Scientific and Virgo Collaborations) Phys. Rev. Lett. 119, 161101 (2017).

[5] F. Kappeler, Rep. Prog. Phys. 52, 945 (1989).

[6] M. S. Smith and K. E. Rehm, Annu. Rev. Nucl. Part. Sci. 51, 91 (2001).

[7] D. L. Burris et al., Astrophys. J. 544, 302 (2000).

[8] C. Sneden et al., Astrophys. J. 467, 819 (1996).

[9] J. Westin et al., Astrophys. J. 530, 783 (2000).

[10] V. Hill et al., Astron. Astrophys. 387, 560 (2002).

[11] A. Bauswein et al., Astrophys. J. Lett. 795, L9 (2014).

[12] S. Shen et al., Astrophys. J. 807, 115 (2015).

[13] J. de Jesús Mendoza-Temis et al., Phys. Rev. C 92, 055805 (2015).

[14] S. Goriely, Phys. Lett. B 436, 10 (1998).

[15] T. Kajino et al., Prog. Part. Nucl. Phys. 107, 109 (2019).

[16] S. Shibagaki, T. Kajino, G. J. Mathews, S. Chiba, S. Nishimura, and G. Lorusso, Astrophys. J. 816, 79 (2016).

[17] M. Terasawa, K. Sumiyoshi, T. Kajino, G. J. Mathews, and I. Tanihata, Astrophys. J. 562, 470 (2001).

[18] G. Baur, C. A. Bertulani, and H. Rebel, Nucl. Phys. A 458, 188 (1986).

[19] U. Datta Pramanik, Prog. Part. Nucl. Phys. 59, 183 (2007).

[20] T. Nakamura, N. Fukuda, N. Aoi, N. Imai, M. Ishihara, H. Iwasaki, T. Kobayashi, T. Kubo, A. Mengoni, T. Motobayashi, M. Notani, H. Otsu, H. Sakurai, S. Shimoura, T. Teranishi, Y. X. Watanabe, and K. Yoneda, Phys. Rev. C 79, 035805 (2009).

[21] H. M. Xu, C. A. Gagliardi, R. E. Tribble, A. M. Mukhamedzhanov, and N. K. Timofeyuk, Phys. Rev. Lett. 73, 2027 (1994).

[22] A. M. Mukhamedzhanov et al., Eur. Phys. J. A 27, 205 (2006).

[23] G. Baur, Phys. Lett. B 178, 135 (1986).

[24] C. Spitaleri, L. Lamia, A. Tumino, R. G. Pizzone, S. Cherubini, A. Del Zoppo, P. Figuera, M. La Cognata, A. Musumarra, M. G. Pellegriti, A. Rinollo, C. Rolfs, S. Romano, and S. Tudisco, Phys. Rev. C 69, 055806 (2004).

[25] W. Younes and H. C. Britt, Phys. Rev. C 67, 024610 (2003).

[26] J. E. Escher, J. T. Burke, F. S. Dietrich, N. D. Scielzo, I. J. Thompson, and W. Younes, Rev. Mod. Phys. 84, 353 (2012).

[27] C. Plettner, H. Ai, C. W. Beausang, L. A. Bernstein, L. Ahle, H. Amro, M. Babilon, J. T. Burke, J. A. Caggiano, R. F. Casten, J. A. Church, J. R. Cooper, B. Crider, G. Gürdal, A. Heinz, E. A. McCutchan, K. Moody, J. A. Punyon, J. Qian, J. J. Ressler, A. Schiller, E. Williams, and W. Younes, Phys. Rev. C 71, 051602(R) (2005).

[28] C. Forssén, F. S. Dietrich, J. Escher, R. D. Hoffman, and K. Kelley, Phys. Rev. C 75, 055807 (2007).

[29] J. A. Cizewski et al., Nucl. Instrumentation B 261, 938 (2007).

[30] R. P. Sánchez et al., Phys. Rev. Lett. 125, 122502 (2020).

[31] A. Spyrou, S. N. Liddick, A. C. Larsen, M. Guttormsen, K. Cooper, A. C. Dombos, D. J. Morrissey, F. Naqvi, G. Perdikakis, S. J. Quinn, T. Renstrøm, J. A. Rodriguez, A. Simon, C. S. Sumithrarachchi, and R. G. T. Zegers, Phys. Rev. Lett. 113, 232502 (2014).

[32] M. Heine et al., Phys. Rev. C 95, 014613 (2017).

[33] M. Roder et al., Phys. Rev. C 93, 065807 (2016).
[34] U. Datta Pramanik et al., Phys. Lett. B 551, 63 (2003).

[35] C. A. Bertulani and G. Baur, Phys. Rep. 163, 299 (1988).

[36] G. Baur, K. Hencken, and D. Trautmann, Prog. Part. Nucl. Phys. 51, 487 (2003).

[37] H. Geissel et al., Nucl. Instrum. Methods Phys. Res. B 70, 286 (1992).

[38] V. Metag et al., Nucl. Phys. A 409, 331c (1983).

[39] J. Cub et al., Nucl. Instrum. Methods Phys. Res. A 402, 67 (1998).

[40] K. Mahata et al., Nucl. Instrum. Methods Phys. Res. A 608, 331 (2009).

[41] T. Blaich et al., Nucl. Instrum. Methods Phys. Res. A 314, 136 (1992).

[42] U. Datta et al., Phys. Rev. C 94, 034304 (2016).

[43] A. Rahaman et al., EPJ Web Conf. 66, 02087 (2014).

[44] C. Caesar et al., Phys. Rev. C 88, 034313 (2013).

[45] R. Palit, P. Adrich, T. Aumann, K. Boretzky, B. V. Carlson, D. Cortina, U. D. Pramanik, T. W. Elze, H. Emling, H. Geissel, M. Hellstrom, K. L. Jones, J. V. Kratz, R. Kulessa, Y. Leifels, A. Leistenschneider, G. Munzenberg, C. Nociforo, P. Reiter, H. Simon, K. Summerer, and W. Walus, Phys. Rev. C 68, 034318 (2003).

[46] C. Nociforo et al., Phys. Lett. B 605, 79 (2005).

[47] T. Aumann and T. Nakamura, Phys. Scr. T152, 014012 (2013).

[48] A. Rahaman et al., J. Phys. G: Nucl. Part. Phys. 44, 045101 (2017).

[49] S. Chakraborty, U. Datta, T. Aumann, S. Beceiro-Novo, K. Boretzky, C. Caesar, B. V. Carlson, W. N. Catford, M. Chartier, D. Cortina-Gil, G. DeAngelis, P. D. Fernandez, H. Emling, O. Ershova, L. M. Fraile, H. Geissel, D. Gonzalez-Diaz, H. Johansson, B. Jonson, N. Kalantar-Nayestanaki, T. Kroll, R. Krucken, C. Langer, T. LeBleis, Y. Leifels, J. Marganiec, G. Munzenberg, M. A. Najafi, T. Nilsson, C. Nociforo, V. Panin, R. Plag, A. Rahaman, R. Reifarth, M. V. Ricciardi, C. Rigollet, D. Rossi, C. Scheidenberger, H. Scheit, H. Simon, J. T. Taylor, Y. Togano, S. Typel, Y. Utsuno, A. Wagner, F. Wamers, H. Weick, and J. S. Winfield, Phys. Rev. C 96, 034301 (2017).

[50] U. Datta et al., in Proceedings of the 13th International Conference on the Intersections of Particle and Nuclear Physics, Palm Spring, California, arXiv:1810.08996v2 (2018).

[51] C. J. Benesh, B. C. Cook, and J. P. Vary, Phys. Rev. C 40, 1198 (1989).

[52] A. Rahaman, Ph.D. thesis, University of Calcutta, Kolkata, West Bengal, Jan. 2018.

[53] S. Chakraborty, Ph.D. thesis, University of Calcutta, Kolkata, West Bengal, Sept. 2016.

[54] K. Boretzky, A. Grünschloß, S. Ilievski, P. Adrich, T. Aumann, C. A. Bertulani, J. Cub, W. Dostal, B. Eberlein, T. W. Elze, H. Emling, M. Fallot, J. Holeczek, R. Holzmann, C. Kozhuharov, J. V. Kratz, R. Kulessa, Y. Leifels, A. Leistenschneider, E. Lubkiewicz, S. Mordechai, T. Ohtsuki, P. Reiter, H. Simon, K. Stelzer, J. Stroth, K. Sümmerer, A. Surowiec, E. Wajda, and W. Walus, Phys. Rev. C 68, 024317 (2003).

[55] www.nndc.bnl.gov.

[56] S. Typel and G. Baur, Phys. Rev. Lett. 93, 142502 (2004).

[57] V. Tripathi, S. L. Tabor, C. R. Hoffman, M. Wiedeking, A. Volya, P. F. Mantica, A. D. Davies, S. N. Liddick, W. F. Mueller, A. Stolz, B. E. Tomlin, T. Otsuka, and Y. Utsuno, Phys. Rev. C 73, 054303 (2006).

[58] F. Rotaru, F. Negoita, S. Grevy, J. Mrazek, S. Lukyanov, F. Nowacki, A. Poves, O. Sorlin, C. Borcea, R. Borcea, A. Buta, 
L. Caceres, S. Calinescu, R. Chevrier, Z. Dombradi, J. M. Daugas, D. Lebhertz, Y. Penionzhkevich, C. Petrone, D. Sohler, M. Stanoiu, and J. C. Thomas, Phys. Rev. Lett. 109, 092503 (2012).

[59] T. Rauscher et al., Astrophys. J. 429, 499 (1994).

[60] T. Rauscher, F. K. Thielemann, and K. L. Kratz, Phys. Rev. C 56, 1613 (1997).

[61] A. L. Sallaska et al., Astrophys. J. Suppl. Ser. 207, 18 (2013).

[62] M. Wiescher et al., J. Phys. G 25, R133 (1999).

[63] H. Herndl, R. Hofinger, J. Jank, H. Oberhummer, J. Gorres, M. Wiescher, F. K. Thielemann, and B. A. Brown, Phys. Rev. C 60, 064614 (1999).

[64] H. Beer, M. Wiescher, F. Käppeler, J. Görres, and P. E. Koehler, Astrophys. J. 387, 258 (1992).

[65] M. Wiescher, J. Görres, and F.-K. Thielemann, Astrophys. J. 363, 340 (1990).

[66] M. Lugaro et al., Astrophys. J. 586, 1305 (2003).

[67] F. Herwig, N. Langer, and M. Lugaro, Astrophys. J. 593, 1056 (2003).
[68] R. Reifarth, M. Heil, C. Forssen, U. Besserer, A. Couture, S. Dababneh, L. Dorr, J. Gorres, R. C. Haight, F. Kappeler, A. Mengoni, S. O'Brien, N. Patronis, R. Plag, R. S. Rundberg, M. Wiescher, and J. B. Wilhelmy, Phys. Rev. C 77, 015804 (2008)

[69] U. D. Pramanik (LAND-FRS Collaboration), Prog. Theor. Phys. Suppl. 146, 427 (2002).

[70] L. Moschini, J. Yang, and P. Capel, Phys. Rev. C 100, 044615 (2019).

[71] N. K. Timofeyuk, D. Baye, P. Descouvemont, R. Kamouni, and I. J. Thompson, Phys. Rev. Lett. 96, 162501 (2006).

[72] M. McCleskey, A. M. Mukhamedzhanov, L. Trache, R. E. Tribble, A. Banu, V. Eremenko, V. Z. Goldberg, Y. W. Lui, E. McCleskey, B. T. Roeder, A. Spiridon, F. Carstoiu, V. Burjan, Z. Hons, and I. J. Thompson, Phys. Rev. C 89, 044605 (2014).

[73] P. Descouvemont, Nucl. Phys. A 675, 559 (2000).

[74] A. Horváth et al., Astrophys. J. 570, 926 (2002).

[75] T. Rauscher and F.-K. Thielemann, At. Data Nucl. Data Tables 75, 1 (2000). 